HRI-P-08-04-001

CU-PHYSICS/08-2008

HRI-RECAPP-08-04

\title{
Exceptional Sensitivity to Neutrino Parameters with a Two-Baseline Beta-Beam Set-up
}

\author{
Sanjib Kumar Agarwalla ${ }^{\star, \dagger, a}$, Sandhya Choubey ${ }^{\star, b}$, Amitava Raychaudhuri ${ }^{\star, \dagger, c}$ \\ ${ }^{\star}$ Harish-Chandra Research Institute, \\ Chhatnag Road, Jhunsi, Allahabad 211019, India \\ ${ }^{\dagger}$ Department of Physics, University of Calcutta, \\ 92 Acharya Prafulla Chandra Road, Kolkata 700009, India
}

\begin{abstract}
We examine the reach of a Beta-beam experiment with two detectors at carefully chosen baselines for exploring neutrino mass parameters. Locating the source at CERN, the two detectors and baselines are: (a) a 50 kton iron calorimeter (ICAL) at a baseline of around $7150 \mathrm{~km}$ which is roughly the magic baseline, e.g., ICAL@INO, and (b) a 50 kton Totally Active Scintillator Detector at a distance of $730 \mathrm{~km}$, e.g., at Gran Sasso. We choose ${ }^{8} \mathrm{~B}$ and ${ }^{8} \mathrm{Li}$ source ions with a boost factor $\gamma$ of 650 for the magic baseline while for the closer detector we consider ${ }^{18} \mathrm{Ne}$ and ${ }^{6} \mathrm{He}$ ions with a range of Lorentz boosts. We find that the locations of the two detectors complement each other leading to an exceptional high sensitivity. With $\gamma=650$ for ${ }^{8} \mathrm{~B} /{ }^{8} \mathrm{Li}$ and $\gamma=575$ for ${ }^{18} \mathrm{Ne} /{ }^{6} \mathrm{He}$ and total luminosity corresponding to $5 \times\left(1.1 \times 10^{18}\right)$ and $5 \times\left(2.9 \times 10^{18}\right)$ useful ion decays in neutrino and antineutrino modes respectively, we find that the two-detector set-up can probe maximal $\mathrm{CP}$ violation and establish the neutrino mass ordering if $\sin ^{2} 2 \theta_{13}$ is $1.4 \times 10^{-4}$ and $2.7 \times 10^{-4}$, respectively, or more. The sensitivity reach for $\sin ^{2} 2 \theta_{13}$ itself is $5.5 \times 10^{-4}$. With a factor of 10 higher luminosity, the corresponding $\sin ^{2} 2 \theta_{13}$ reach of this set-up would be $1.8 \times 10^{-5}$, $4.6 \times 10^{-5}$ and $5.3 \times 10^{-5}$ respectively for the above three performance indicators. CP violation can be discovered for $64 \%$ of the possible $\delta_{\mathrm{CP}}$ values for $\sin ^{2} 2 \theta_{13} \geq 10^{-3}\left(\geq 8 \times 10^{-5}\right)$, for the standard luminosity (10 times enhanced luminosity). Comparable physics performance can be achieved in a set-up where data from CERN to INO@ICAL is combined with that from CERN to the Boulby mine in United Kingdom, a baseline of $1050 \mathrm{~km}$.
\end{abstract}

${ }^{a}$ email: sanjib@hri.res.in

${ }^{b}$ email: sandhya@hri.res.in

${ }^{c}$ email: raychaud@hri.res.in 


\section{Introduction}

Long baseline experiments with powerful neutrino beams $[1,2,3,4]$ from upgraded accelerator facilities are the next frontier in neutrino oscillation physics. Set-ups with intense neutrino beam sources and smart detector technologies are being planned to fathom the hitherto uncharted regimes of the neutrino mass matrix. In particular, the long baseline experiments are being designed to measure the third mixing angle $\theta_{13}$, CP phase $\delta_{\mathrm{CP}}$ and $\operatorname{sgn}\left(\Delta m_{31}^{2}\right)$ aka, the neutrino mass hierarchy ${ }^{1}$. The most promising avenue for this purpose is the $\nu_{e} \rightarrow \nu_{\mu}$ oscillation channel $P_{e \mu}$ (or its T-conjugate $P_{\mu e}$ ), often referred to in the literature as the "golden channel" [5]. It was realized that while this channel can be used in the most cost-effective way to measure all three neutrino oscillation parameters listed above, it is also rife with the so-called problem of "parameter degeneracies". These are identified as the $\left(\theta_{13}, \delta_{\mathrm{CP}}\right)$ intrinsic degeneracy [6], the $\left(\operatorname{sgn}\left(\Delta m_{31}^{2}\right), \delta_{\mathrm{CP}}\right)$ degeneracy [7], and the $\left(\theta_{23}, \pi / 2-\theta_{23}\right)$ degeneracy [8]. Together they could result in as many as eight-fold degenerate solutions [9], of which, obviously, only one is true. This is evidently a very undesirable situation and a large body of existing literature is devoted to finding ways of combating this menace $[6,10,11,12,13,14,15,16,17]$. A particularly attractive way of killing the clone solutions from the $\delta_{\mathrm{CP}}$ dependent degeneracies is to perform the experiment at the "magic baseline" $[18,19,20]$. At this baseline all $\delta_{\mathrm{CP}}$ dependent terms drop out, providing an ideal bedrock for measuring $\theta_{13}$ and $\operatorname{sgn}\left(\Delta m_{31}^{2}\right)$.

In $[21,22,23]$ we expounded the sensitivity reach of a magic baseline experimental set-up where the neutrino source is a Beta-beam [24] at CERN and the detector is a large magnetized iron calorimeter (ICAL) at the India-based Neutrino Observatory ${ }^{2}$ (INO) [25]. Using a 50 kton fiducial mass and $80 \%$ detector efficiency ${ }^{3}$ for ICAL@INO, and considering a Beta-beam using ${ }^{8} \mathrm{~B}$ and ${ }^{8} \mathrm{Li}$ as ion source [26, 27], with a Lorentz boost $\gamma=650$ and assuming $5 \times\left(1.1 \times 10^{18}\right)$ and $5 \times\left(2.9 \times 10^{18}\right)$ useful ion decays in neutrino and antineutrino modes respectively, we showed that this set-up could unambiguously probe $\operatorname{sgn}\left(\Delta m_{31}^{2}\right)$ at $3 \sigma$ if ${ }^{4} \sin ^{2} 2 \theta_{13}$ (true) $>5.6 \times 10^{-4}$ and find a signal for $\theta_{13}$ at the same level of significance if $\sin ^{2} 2 \theta_{13}$ (true) $>5.1 \times 10^{-4}$, independent of the true neutrino mass hierarchy and $\delta_{\mathrm{CP}}$ (true). The $\theta_{13}$ and hierarchy sensitivity reach of this "magical" set-up is therefore superior to that of most other rival proposals involving Beta-beams $[16,28,29,30,31,32,33,34,35,36,37,38,39]$, and is almost comparable to that possible with a Neutrino Factory [40] at the magic baseline. This tremendous sensitivity of the CERN-INO Beta-beam project to $\theta_{13}$ and hierarchy comes from a combination of being close to the magic baseline as well as from being sensitive to near-maximal matter effects.

The main drawback of the CERN-INO Beta-beam set-up is its inability to determine $\delta_{\mathrm{CP}}$, since being almost at the magic baseline it is insensitive to the CP phase. Note that this is also true for the Neutrino Factory experiment at the magic baseline. This is why the optimal Neutrino Factory set-up demands a combination of two baselines, one magic and the other around $L=4000 \mathrm{~km}$, where one has the best sensitivity to $\delta_{\mathrm{CP}}[40]$. For Beta-beams, one has to optimize not only

\footnotetext{
${ }^{1}$ In this paper we define $\Delta m_{i j}^{2}=m_{i}^{2}-m_{j}^{2}$ and refer to $\operatorname{sgn}\left(\Delta m_{31}^{2}\right)$ as the neutrino mass hierarchy $-\operatorname{sgn}\left(\Delta m_{31}^{2}\right)>$ 0 is called "normal hierarchy" $(\mathrm{NH})$ while $\operatorname{sgn}\left(\Delta m_{31}^{2}\right)<0$ is called "inverted hierarchy" (IH). We stress that the arguments are valid for both hierarchical as well as quasi-degenerate neutrino mass spectra.

${ }^{2}$ The CERN to INO distance is $7152 \mathrm{~km}$, which is tantalizingly close to the magic baseline.

${ }^{3}$ We give details about the detector configuration in section 3 .

${ }^{4}$ These are the values of the parameters chosen by Nature, to be distinguished from the fitted values. Throughout this paper we denote the true value of a parameter by putting "(true)" after the symbol for the parameter.
} 
on $\gamma$, the baseline $L$, and the luminosity, but also on the choice of the source ions. A detailed optimization study has revealed that for intermediate values of $\gamma$, one would preferentially use ${ }^{8} \mathrm{~B}$ and ${ }^{8} \mathrm{Li}$ at the magic baseline for the mass hierarchy, ${ }^{18} \mathrm{Ne}$ and ${ }^{6} \mathrm{He}$ at an intermediate baseline of $L=600-900 \mathrm{~km}$ for $\mathrm{CP}$ violation, and either ${ }^{18} \mathrm{Ne}$ and ${ }^{6} \mathrm{He}$ at the intermediate baseline or ${ }^{8} \mathrm{~B}$ and ${ }^{8} \mathrm{Li}$ at the magic baseline for the $\sin ^{2} 2 \theta_{13}$ discovery [41].

In this paper we consider a two-baseline Beta-beam set-up, one with $L=7152 \mathrm{~km}$, the CERNINO baseline, and another with $L=730 \mathrm{~km}$ which is the CERN-Gran Sasso (LNGS) distance. For the CERN-INO case ${ }^{8} \mathrm{~B}$ and ${ }^{8} \mathrm{Li}$ are the preferred source ions and we take $\gamma=650$. For the CERN-LNGS set-up, on the other hand, we choose the ${ }^{18} \mathrm{Ne}$ and ${ }^{6} \mathrm{He}$ ions and allow their $\gamma$ to vary between $250-650^{5}$ in order to examine the dependence of the sensitivity on the value of the boost factor. Since the ${ }^{8} \mathrm{~B}$ and ${ }^{8} \mathrm{Li}$ ions would produce multi-GeV neutrino beams for $\gamma=650$, we use a 50 kton iron calorimeter (ICAL) for the longer baseline at INO and call this set-up CERN-ICAL@INO. For the intermediate baseline option, since we are interested in the lower energy ${ }^{18} \mathrm{Ne}$ and ${ }^{6} \mathrm{He}$ ions, we assume a 50 kton Totally Active Scintillator Detector (TASD) in order to harness the low energy events required for better CP sensitivity. We present results for $5 \times\left(1.1 \times 10^{18}\right)$ and $5 \times\left(2.9 \times 10^{18}\right)$ useful ion decays in neutrino and antineutrino modes respectively for both baseline set-ups. We also show the projected sensitivity if one achieves statistics that are larger by a factor of 10 . Since the statistics depend on the product of the size of the detector, the exposure time, detector efficiency and the number of useful ion decays, this one order of magnitude increase could come from a combination of enhanced performance in any of the factors mentioned above. In particular, it might be possible to have 10 times larger useful ion decays per year in the storage ring [43]. ICAL@INO could also be upgraded to $100 \mathrm{kton}$.

Note that while most of our figures and discussion in the text would explicitly be for the CERN-LNGS intermediate baseline set-up, we also present results for the case where a ${ }^{18} \mathrm{Ne}$ and ${ }^{6} \mathrm{He}$ Beta-beam is shot from CERN to the Boulby mine in the United Kingdom (see [38] and references therein). The CERN-Boulby distance is $1050 \mathrm{~km}$, and one can check from Fig. 5 of [41] that the CP sensitivity for this baseline is only marginally weaker than that for $L=730$ $\mathrm{km}$. Therefore, when combined with the CERN-INO data, we expect similar performance for this baseline option as well.

In some sense this work is a part of an ongoing international exercise in sharpening the full capability of long baseline Beta-beam experiments to explore neutrino properties. In addition to the work discussed earlier in the Introduction, some of the milestones on this route can be identified as the CERN-MEMPHYS proposal [16, 28, 29], its variants with higher $\gamma[32,33]$, using a cocktail of sources [37], detector options, etc.

While the best $\mathrm{CP}$ violation sensitivity comes with ${ }^{18} \mathrm{Ne}$ and ${ }^{6} \mathrm{He}$ as source at our chosen intermediate baseline, it might be more convenient and less demanding to use the same set of ions at both the baselines. The optimal baseline for $\mathrm{CP}$ studies with ${ }^{8} \mathrm{~B}$ and ${ }^{8} \mathrm{Li}$ was seen to be around 1000-2000 km in [41]. In [31], the authors proposed a set-up with this scenario where they

\footnotetext{
${ }^{5}$ With the existing facilities at CERN, one will be able to accelarate ${ }^{6} \mathrm{He}$ to only about $\gamma=150$. This corresponds to a boost factor of about 250 for ${ }^{18} \mathrm{Ne}$ and 280 for ${ }^{8} \mathrm{~B}$, due to the different charge to mass ratios for these ions. However, with the "Super-SPS", an upgraded version of the SPS with super-conducting magnets [33, 30] it should be possible to accelarate ${ }^{6} \mathrm{He}$ to $\gamma=350$, which corresponds to $\gamma=575$ for ${ }^{18} \mathrm{Ne}$ and $\gamma=650$ for ${ }^{8} \mathrm{~B}$. The Tevatron at Fermilab could produce a Beta-beam with similar boost factors. Higher acceleration at CERN would require the use of the LHC itself, with $\gamma>1000$ possible [42].
} 
considered a two-baseline combination with a longer $L \simeq 7000 \mathrm{~km}$ and a shorter $L \simeq 2000 \mathrm{~km}$. A $\gamma=350$ Beta-beam produced by ${ }^{8} \mathrm{~B}$ and ${ }^{8} \mathrm{Li}$ as source ions feeds both detectors and they consider up to $5 \times\left(10 \times 10^{18}\right)$ total useful ion decays in both neutrino and antineutrino modes. They consider 50 kton of magnetized iron as the end detector for both baselines. In this set-up the mass hierarchy can be discovered if $\sin ^{2} 2 \theta_{13}$ (true) $>3 \times 10^{-4}$ and CP violation can be established for $70 \%$ of the possible $\delta_{\mathrm{CP}}$ (true) values if $\sin ^{2} 2 \theta_{13}$ (true) $>10^{-3}$. Signal for the mixing angle itself could be observed for $\sin ^{2} 2 \theta_{13}$ (true) $>1 \times 10^{-4}$. For $5 \times\left(2 \times 10^{18}\right)$ total useful decays, which is comparable to our standard luminosity, the corresponding reaches are $\sin ^{2} 2 \theta_{13}$ (true) $\gtrsim 10^{-3}$ for the mass hierarchy, and $\sin ^{2} 2 \theta_{13}$ (true) $\gtrsim 8 \times 10^{-4}$ for discovering $\theta_{13}$. CP violation sensitivity gets severely restricted and deteriorates, especially for $\delta_{\mathrm{CP}}$ (true) $=270^{\circ}$. The poor $\mathrm{CP}$ reach for lower luminosity comes due to the choice of ${ }^{8} \mathrm{~B}$ and ${ }^{8} \mathrm{Li}$ as source ions (see [41] for a detailed discussion). Since much better CP sensitivity for plausible luminosity and $\gamma$ can be achieved with the ${ }^{18} \mathrm{Ne}$ and ${ }^{6} \mathrm{He}$ combination, we will exclusively use them for our intermediate baseline option of CERN to LNGS. Since our chosen $\gamma$ is larger, we also achieve sensitivity to mass hierarchy and $\theta_{13}$ which is better by at least an order of magnitude for the same number of useful ions decays.

The paper is organized as follows. In the next section we briefly discuss the oscillation probability for the golden channel and highlight the magic baseline behavior. The experimental set-up which we consider is outlined in the subsequent section. In the following section we explore the prospects of the TASD@LNGS detector, in a stand-alone mode, for exploring CP violation. This is to set the stage for the next section where the results of a combined two-baseline analysis are presented with emphasis on the sensitivities to $\mathrm{CP}$ violation, $\theta_{13}$, and the neutrino mass hierarchy. We end by summarizing the results for our combined CERN-INO and CERN-LNGS set-ups and make a comparative discussion of this set-up vis-a-vis the combined CERN-INO and CERNBoulby set-up. We also compare our set-up against the one studied in [31] and discuss the pros and cons of the two proposals. Some remarks about the beam related as well as atmospheric neutrino backgrounds are collected in the Appendix.

\section{Golden channel oscillations}

For the results presented in this paper we have calculated the neutrino oscillation probability in matter exactly, using the PREM profile for the Earth matter density [44]. However, for elucidating the behavior of neutrino oscillations as a function of baseline and/or neutrino energy, it is useful to exploit the approximate analytic formula for the golden channel probability $P_{e \mu}$ in matter $[45$, $46,47]$, keeping terms only up to second order in the small quantities $\theta_{13}$ and $\alpha \equiv \Delta m_{21}^{2} / \Delta m_{31}^{2}$ $[5,48]$

$$
\begin{aligned}
P_{e \mu} & \simeq \sin ^{2} \theta_{23} \sin ^{2} 2 \theta_{13} \frac{\sin ^{2}[(1-\hat{A}) \Delta]}{(1-\hat{A})^{2}} \\
& \pm \alpha \sin 2 \theta_{13} \sin 2 \theta_{12} \sin 2 \theta_{23} \sin \delta_{\mathrm{CP}} \sin (\Delta) \frac{\sin (\hat{A} \Delta)}{\hat{A}} \frac{\sin [(1-\hat{A}) \Delta]}{(1-\hat{A})} \\
& +\alpha \sin 2 \theta_{13} \sin 2 \theta_{12} \sin 2 \theta_{23} \cos \delta_{\mathrm{CP}} \cos (\Delta) \frac{\sin (\hat{A} \Delta)}{\hat{A}} \frac{\sin [(1-\hat{A}) \Delta]}{(1-\hat{A})}
\end{aligned}
$$




$$
+\alpha^{2} \cos ^{2} \theta_{23} \sin ^{2} 2 \theta_{12} \frac{\sin ^{2}(\hat{A} \Delta)}{\hat{A}^{2}}
$$

where

$$
\Delta \equiv \frac{\Delta m_{31}^{2} L}{4 E}, \quad \hat{A} \equiv \frac{A}{\Delta m_{31}^{2}}, \quad A= \pm 2 \sqrt{2} G_{F} N_{e} E
$$

Above, $A$ is the matter potential, expressed in terms of the electron density $N_{e}$ and the (anti)neutrino energy $E$; the ' + ' sign refers to neutrinos while the ' - ' to antineutrinos.

From Eq. (1) it is apparent that at a baseline for which

$$
\sin (\hat{A} \Delta) \simeq 0
$$

the oscillation probability is insensitive to $\delta_{\mathrm{CP}}$. This defines the "magic baseline". As noted, the CERN-INO distance closely matches this baseline and it has been shown in earlier work [21, 22, 23] that this ensures a high sensitivity at the same time to the determination of $\theta_{13}$ and the extraction of the neutrino mass hierarchy. In this work, we examine the benefits which accrue from combining two Beta-beam experiments both with the source at CERN, one with the detector at INO and the other located at an appropriate distance where the impact of $\mathrm{CP}$ violation is prominent. Taking a cue from an optimization analysis of such Beta-beam set-ups [41], this second detector is assumed to be at Gran Sasso.

We will refer to Eqs. (1) and (2) from time to time to identify the physics underlying the results which we present later.

\section{The Experimental Set-up}

The number of (anti)muon events in the $i$ th energy bin in the detector is given by

$$
N_{i}=\frac{T n_{n} f_{I D} \epsilon}{4 \pi L^{2}} \int_{0}^{E_{\max }} d E \int_{E_{A_{i}}^{\min }}^{E_{A_{i}}^{\max }} d E_{A} \phi(E) \sigma_{\nu_{\mu}}(E) R\left(E, E_{A}\right) P_{e \mu}(E),
$$

where $T$ is the total running time, $n_{n}$ is the number of target nucleons in the detector, $f_{I D}$ is the charge identification efficiency (meaningful only for the magnetized iron detector), $\epsilon$ is the detector efficiency and $R\left(E, E_{A}\right)$ is the energy resolution function of the detector, which we assume is a Gaussian. For muon (antimuon) events, $\sigma_{\nu_{\mu}}$ is the neutrino (antineutrino) interaction cross-section. The quantities $E$ and $E_{A}$ are the true and reconstructed (anti)neutrino energies respectively. The quantity $\phi(E)$ is the (anti)neutrino Beta-beam flux produced at the source, with Lorentz boost $\gamma$ and $L$ is the baseline.

\subsection{The Source Ions}

The technological challenge for producing the Beta-beam involves creating, bunching, accelerating and storing beta unstable radioactive ions $[49,50]$. A pure and intense $\nu_{e}$ and/or $\bar{\nu}_{e}$ beam is produced when these highly accelerated ions decay along the straight sections of the storage ring. 


\begin{tabular}{||c||c||c||c||c||c||}
\hline \hline Ion & $\tau(\mathrm{s})$ & $E_{0}(\mathrm{MeV})$ & $f$ & Decay fraction & Beam \\
\hline${ }_{10}^{18} \mathrm{Ne}$ & 2.41 & 3.92 & 820.37 & $92.1 \%$ & $\nu_{e}$ \\
${ }_{2}^{6} \mathrm{He}$ & 1.17 & 4.02 & 934.53 & $100 \%$ & $\bar{\nu}_{e}$ \\
\hline${ }_{5}^{8} \mathrm{~B}$ & 1.11 & 14.43 & 600872.07 & $100 \%$ & $\nu_{e}$ \\
${ }_{3}^{8} \mathrm{Li}$ & 1.20 & 13.47 & 425355.16 & $100 \%$ & $\bar{\nu}_{e}$ \\
\hline \hline
\end{tabular}

Table 1: Beta decay parameters: lifetime $\tau$, electron total end-point energy $E_{0}, f$-value and decay fraction for various ions [51].

The choice of ions is governed by a number of factors which include their end-point energy, lifetime, production rate and their charge to mass ratios. Two sets of source ions have been identified as plausible candidates for the production of a Beta-beam. The most widely discussed ions are ${ }^{18} \mathrm{Ne}$ and ${ }^{6} \mathrm{He}$ for $\nu_{e}$ and $\bar{\nu}_{e}$ beam respectively. The alternative set of ions which have also been considered extensively in the recent literature are ${ }^{8} \mathrm{~B}$ and ${ }^{8} \mathrm{Li}$ for $\nu_{e}$ and $\bar{\nu}_{e}$ beam respectively. We present in Table 1 the relevant details of the properties of these ions. We note that the main difference between the two sets lies in their total end-point energies $-{ }^{8} \mathrm{~B}\left({ }^{8} \mathrm{Li}\right)$ has an end-point energy, $E_{0}$, which is 3.68 (3.35) times that of ${ }^{18} \mathrm{Ne}\left({ }^{6} \mathrm{He}\right)$. The Beta-beam flux spectrum depends solely on $\gamma$ and $E_{0}$ and the energy of the beam is often crucial in determining the type of physics that can be explored. We therefore reiterate the following points [41, 4]:

- Since the peak neutrino energy is roughly given as $\gamma E_{0}$, the boost factor needed for ${ }^{18} \mathrm{Ne}\left({ }^{6} \mathrm{He}\right)$ should be about 3.68 (3.35) times that needed by ${ }^{8} \mathrm{~B}\left({ }^{8} \mathrm{Li}\right)$ in order to achieve the same neutrino peak energy.

- Since the total number of neutrinos peaked in the forward direction, i.e., towards the detector, roughly goes as $\gamma^{2}$, larger $\gamma$ enhances the flux. Higher boost factors are therefore preferred.

- The reference number of useful decays which we use for antineutrinos $\left(2.9 \times 10^{18}\right.$ per year $)$ is larger than that for neutrinos $\left(1.1 \times 10^{18}\right.$ per year $)$. The ratio between the two just about compensates for the smaller antineutrino interaction cross section in matter. So, roughly, this results in a symmetry between the $\nu$ and $\bar{\nu}$ data.

We have seen in $[22,23]$ that the higher end-point energy of ${ }^{8} \mathrm{~B}$ and ${ }^{8} \mathrm{Li}$ allows these ions to produce Beta-beams with peak energy in the multi-GeV regime, where one obtains near-resonant matter effects for the near-magic baseline. This can be achieved with plausible values of $\gamma$, and results in remarkable sensitivity to $\theta_{13}$ and $\operatorname{sgn}\left(\Delta m_{31}^{2}\right)$. Indeed this set of ions emerged as the preferred choice for the magic baseline after a thorough scan of plausible $\gamma$ values [41]. As mentioned before, the $\delta_{\mathrm{CP}}$ sensitivity is smothered at the magic baseline. It was seen in [41] that intermediate baselines are far superior for CP studies. It was further noted that CP sensitivity with ${ }^{18} \mathrm{Ne}$ and ${ }^{6} \mathrm{He}$ is much better than that with ${ }^{8} \mathrm{~B}$ and ${ }^{8} \mathrm{Li}$. Therefore, we take ${ }^{8} \mathrm{~B}$ and ${ }^{8} \mathrm{Li}$ as source ions for the CERN-INO baseline and ${ }^{18} \mathrm{Ne}$ and ${ }^{6} \mathrm{He}$ as the source ions for the CERN-LNGS baseline. We will fix the Lorentz boost of ${ }^{8} \mathrm{~B}$ and ${ }^{8} \mathrm{Li}$ for CERN-INO as $\gamma=650$ and will study the impact of $\gamma$ for ${ }^{18} \mathrm{Ne}$ and ${ }^{6} \mathrm{He}$ at the shorter baseline. 


\begin{tabular}{||c||c||c||}
\hline \hline Detector Characteristics & $\begin{array}{c}\text { ICAL@INO } \\
\left(\text { Only } \mu^{ \pm}\right)\end{array}$ & $\begin{array}{c}\text { TASD@LNGS } \\
\left(\text { Both } \mu^{ \pm} \& e^{ \pm}\right)\end{array}$ \\
\hline \hline Total Mass & $50 \mathrm{kton}$ & $50 \mathrm{kton}$ \\
\hline Energy threshold & $1 \mathrm{GeV}$ & $0.5 \mathrm{GeV}$ \\
\hline Detection Efficiency $(\epsilon)$ & $80 \%$ & $80 \%\left(\mu^{ \pm}\right) \& 20 \%\left(e^{ \pm}\right)$ \\
\hline Charge Identification Efficiency $\left(f_{I D}\right)$ & $95 \%$ & No CID \\
\hline Energy Resolution $(\sigma)(\mathrm{GeV})$ & $0.15 \mathrm{E}(\mathrm{GeV})$ & $\begin{array}{c}0.03 \sqrt{\mathrm{E}(\mathrm{GeV})} \text { for } \mu^{ \pm} \\
0.06 \sqrt{\mathrm{E}(\mathrm{GeV})} \text { for } e^{ \pm}\end{array}$ \\
\hline Bin Size & $1 \mathrm{GeV}$ & $0.2 \mathrm{GeV}$ \\
\hline Background Rejection & 0.0001 & 0.001 \\
\hline Signal error & $2.5 \%$ & $2.5 \%$ \\
\hline Background error & $5 \%$ & $5 \%$ \\
\hline \hline
\end{tabular}

Table 2: Detector characteristics used in the simulations. The bin size is kept fixed, while the number of bins is varied according to the maximum energy.

Throughout this work we deal with results obtained by combining data from neutrino and antineutrino beams. Further, we ascribe the same Lorentz boost $\gamma$ to both beams. It has to be borne in mind that the charge to mass ratios, $(Z / A)$, of the source ions are not the same (see Table 1), and so the same boost cannot be achieved if the ions run simultaneously in a ring. Note, however,

$$
\left(\frac{Z}{A}\right)_{{ }_{8 \mathrm{~B}}}:\left(\frac{Z}{A}\right)_{{ }_{18 \mathrm{Ne}}}=\left(\frac{Z}{A}\right)_{{ }_{8 \mathrm{Li}}}:\left(\frac{Z}{A}\right)_{{ }^{6} \mathrm{He}}=9: 8
$$

Consequently, if the two neutrino (antineutrino) source ions are run simultaneously in the ring in which the ${ }^{8} \mathrm{~B}\left({ }^{8} \mathrm{Li}\right)$ ions have a Lorentz boost $\gamma=650$, then the ${ }^{18} \mathrm{Ne}\left({ }^{6} \mathrm{He}\right)$ source ions will have a $\gamma$ of 575 . Of course, these are but sample boost factors for illustrating the feature that simultaneous run configurations are possible for the chosen source ions, with the proviso that the $\left({ }^{8} \mathrm{~B}\right.$ and $\left.{ }^{8} \mathrm{Li}\right)$ pair have a different value of $\gamma$ related to that of the $\left({ }^{18} \mathrm{Ne}\right.$ and $\left.{ }^{6} \mathrm{He}\right)$ pair.

\subsection{The ICAL@INO detector}

The ICAL detector at INO will be a 50 kton magnetized iron calorimeter with Resistive Plate Chambers (RPCs) serving as the active elements [25]. The first phase of this detector (starting around 2012) will focus on atmospheric neutrino measurements. The entire ICAL detector will be split into 3 modules, each of which would be of dimension $16 \mathrm{~m} \times 16 \mathrm{~m} \times 12 \mathrm{~m}$. The detector structure consists of iron layers of $6 \mathrm{~cm}$ thickness with a $2 \mathrm{~cm}$ gap between layers wherein the glass RPCs will be interleaved. The detector would be magnetized by a field of about 1 Tesla. The detector characteristics that we have used for our simulation are shown in Table 2. It is planned that ICAL@INO will be upgraded to 100 kton in the future. Number of expected muon (or antimuon) events can be calculated using Eq. (4). 

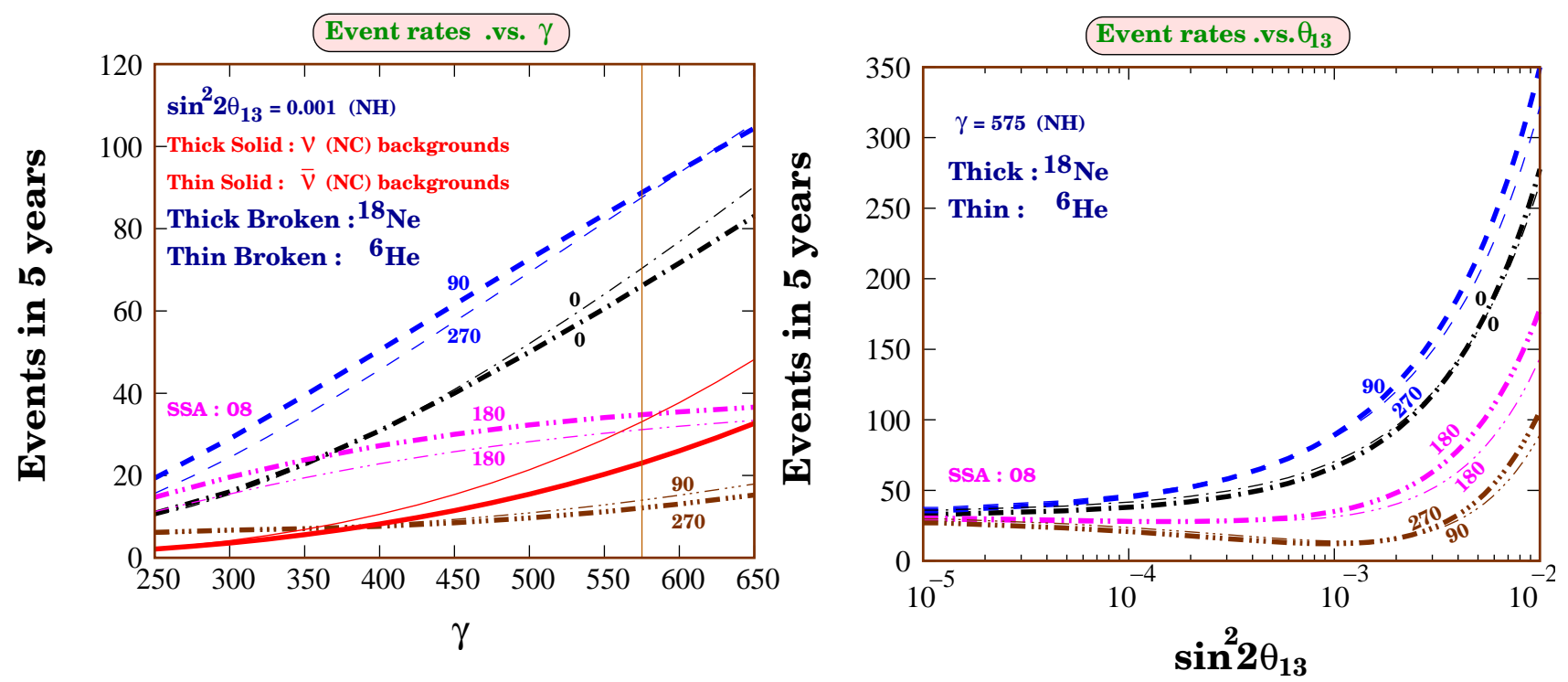

Figure 1: Total event rates expected in 5 years at the CERN-TASD@LNGS set-up assuming $1.1 \times 10^{18}$ and $2.9 \times 10^{18}$ useful decays per year for ${ }^{18} \mathrm{Ne}$ and ${ }^{6} \mathrm{He}$, respectively. The left panel shows the dependence on the boost factor at $\sin ^{2} 2 \theta_{13}=10^{-3}$, while the right panel depicts the variation with $\sin ^{2} 2 \theta_{13}$ for a fixed $\gamma=500$. Results are shown for four values of $\delta_{\mathrm{CP}}: 0^{\circ}, 90^{\circ}, 180^{\circ}$, and $270^{\circ}$ for both neutrinos (thick broken lines) and antineutrinos (thin broken lines). Normal hierarchy has been assumed. In the left panel, the vertical line corresponds to $\gamma=575$ (see text) and the solid curves are the effective neutral current backgrounds for neutrinos (thick red lines) and antineutrinos (thin red lines).

\subsection{The TASD@LNGS detector}

For the CERN-LNGS baseline we will be working with a 50 kton TASD. A scintillator detector has several virtues. It has good detection efficiency for muons, can support a low energy threshold and has excellent energy resolution. It can also detect electrons but with a lower efficiency. On the flip side, unlike in the iron calorimeter, there is no charge identification. The detector characteristics we have assumed $[2,35]$ are listed in Table 2. One can see from the Table that the background suppression capability of a TASD is also expected to be one order poorer than that of an iron calorimeter detector ${ }^{6}$. The effect of this on the results is discussed later. However, the biggest advantage of TASD over iron calorimeters is its much lower threshold energy. This helps in eliminating the clone solutions and hence delivers better CP sensitivity.

We begin with some remarks about the performance of the CERN-TASD@LNGS Beta-beam set-up in isolation before turning to our main theme, namely, a combined analysis of the results from two detectors at different baselines. The number of useful ion decays that we choose as reference values are $\left(1.1 \times 10^{18}\right)$ per year for ${ }^{18} \mathrm{Ne}$ and $\left(2.9 \times 10^{18}\right)$ per year for ${ }^{6} \mathrm{He}$. For comparison we also present results obtained using luminosities one order higher than these reference values. We combine data from $\nu$ and $\bar{\nu}$ runs, and assume that both are at the same Lorentz boost $\gamma$. The

\footnotetext{
${ }^{6}$ This can be traced to the fact that in scintillator detectors hadrons travel farther before they stop or decay, enhancing the possibility that they mimic the signal. The lack of charge identification is also a handicap in this regard.
} 
values of the oscillation parameters and the earth's matter density profile that we use are listed in Table 3.

In Fig. 1 the five-year (anti)muon event rates for the TASD detector at Gran Sasso are shown. In the left panel the number of events is shown as a function of $\gamma$ for $\sin ^{2} 2 \theta_{13}=10^{-3}$ while in the right panel it is shown as a function of $\sin ^{2} 2 \theta_{13}$ when $\gamma$ is fixed at 575 . In both panels results are shown for both $\nu$ and $\bar{\nu}$ beams. Normal neutrino mass hierarchy has been assumed. Notice that in both panels, to a good approximation, the number of events for neutrinos for any $\delta_{\mathrm{CP}}$ closely matches that for antineutrinos but with $\mathrm{CP}$ phase $-\delta_{\mathrm{CP}}$. This behavior can be readily understood from Eq. (1). At the CERN-LNGS shorter baseline, matter effects are small and hence $\hat{A}<<1$ in Eq. (2). It follows that the $\delta_{\mathrm{CP}}$ dependence of the probabilities can be expressed as $P_{e \mu} \simeq A_{0}+A_{-} \sin \delta_{\mathrm{CP}}+A_{+} \cos \delta_{\mathrm{CP}}$ and $P_{\bar{e} \bar{\mu}} \simeq A_{0}-A_{-} \sin \delta_{\mathrm{CP}}+A_{+} \cos \delta_{\mathrm{CP}}$, where $A_{0}, A_{ \pm}$ are independent of $\delta_{\mathrm{CP}}$, whence the symmetry is manifest. In the left panel, the increase in the number of events with increasing Lorentz boost $\gamma$ is a consequence of the resultant enhancement of the on-axis flux as well as the higher energy of the beam. The reason why neutrino event rates are higher for $\delta_{\mathrm{CP}}=90^{\circ}$ and $0^{\circ}$ compared to that for $\delta_{\mathrm{CP}}=180^{\circ}$ and $270^{\circ}$ can be easily seen from the above expressions. One can explicitly check that $A_{+}$and $A_{-}$are positive, which means that for $\delta_{\mathrm{CP}}=180^{\circ}$ and $270^{\circ}$ number of events are given by a difference of two positive terms while for $\delta_{\mathrm{CP}}=90^{\circ}$ and $0^{\circ}$ they come as the corresponding sum. The reason why the slope of the curves for $\delta_{\mathrm{CP}}=180^{\circ}$ and $\delta_{\mathrm{CP}}=270^{\circ}\left(90^{\circ}\right)$ for neutrinos (antineutrinos) is relatively smaller than that of $\delta_{\mathrm{CP}}=0^{\circ}$ and $\delta_{\mathrm{CP}}=90^{\circ}\left(270^{\circ}\right)$ for neutrinos (antineutrinos) can be understood from a similar reasoning. The behavior of the number of events with $\sin ^{2} 2 \theta_{13}$ (right panel) is not difficult to explain. As $\sin ^{2} 2 \theta_{13}$ increases from a near-zero value, first the 2 nd and 3rd terms in Eq. (1), which are linear in $\sin 2 \theta_{13}$ and are dependent on $\delta_{\mathrm{CP}}$, begin to contribute. Beyond a certain point, the first term takes over leading to the increasing behavior for all curves in the right panel.

For the purpose of illustration, in the left panel of Fig. 1 we also present the $\nu$ and $\bar{\nu} \mathrm{NC}$ background estimates. Notice that the background is quite significant for the entire range of $\gamma$. We have also indicated the point corresponding to $\gamma=575$, which, as discussed earlier, is the $\gamma$ for ${ }^{18} \mathrm{Ne}\left({ }^{6} \mathrm{He}\right)$ ions when they are run in the same storage ring as ${ }^{8} \mathrm{~B}\left({ }^{8} \mathrm{Li}\right)$ with the $\gamma$ for the latter being 650 .

Note that in our analysis for this detector, we have also included the information from the $P_{e e}$ and $P_{\bar{e} \bar{e}}$ channels, albeit with the reduced detector efficiency (see Table 2) of only $20 \%$ for electrons. The number of electron events can be calculated using Eq. (4), by making appropriate changes to the oscillation probability and cross-sections. However, as was noted in [13, 14], this channel has hardly any sensitivity to $\theta_{13}$ and mass ordering at low baselines like the one under discussion. It is independent of $\delta_{\mathrm{CP}}$.

\section{CP sensitivity: CERN-TASD@LNGS Set-up}

The goal of this work is to explore the power of a two-detector Beta-beam experiment to unravel neutrino mass parameters. The detector at the magic baseline is insensitive to the CP phase $\delta_{\mathrm{CP}}$ and so the main emphasis of the closer detector will be to address this issue. To set the stage for the full two-detector analysis, in this section we discuss the CERN-TASD@LNGS combination as a stand-alone set-up for exploring CP violation. 


\begin{tabular}{||c||c||}
\hline \hline Benchmark Values & $1 \sigma$ estimated error \\
\hline \hline $\mid \Delta m_{31}^{2}($ true $) \mid=2.5 \times 10^{-3} \mathrm{eV}^{2}$ & $\sigma\left(\left|\Delta m_{31}^{2}\right|\right)=1.5 \%$ \\
\hline $\sin ^{2} 2 \theta_{23}($ true $)=1.0$ & $\sigma\left(\sin ^{2} 2 \theta_{23}\right)=1 \%$ \\
\hline$\Delta m_{21}^{2}($ true $)=8.0 \times 10^{-5} \mathrm{eV}^{2}$ & $\sigma\left(\Delta m_{21}^{2}\right)=2 \%$ \\
\hline $\sin ^{2} \theta_{12}($ true $)=0.31$ & $\sigma\left(\sin ^{2} \theta_{12}\right)=6 \%$ \\
\hline$\rho($ true $)=1(\mathrm{PREM})$ & $\sigma(\rho)=5 \%$ \\
\hline \hline
\end{tabular}

Table 3: Chosen benchmark values of the oscillation parameters and the earth matter density profile and their $1 \sigma$ estimated error.

The $3 \sigma$ sensitivity to "maximal CP violation" is presented in Fig. 2 as a function of the boost factor $\gamma$ : these are the (red) solid curves marked "CG" in both panels. This performance indicator is defined as follows. We generate the data for $\delta_{\mathrm{CP}}$ (true) $=90^{\circ}$ (left panel) or $\delta_{\mathrm{CP}}($ true) $=$ $270^{\circ}$ (right panel), while in theory we allow $\delta_{\mathrm{CP}}$ to be $0^{\circ}$ and $180^{\circ}$. We marginalize over the normalization of the PREM density profile and all other oscillation parameters, including the neutrino mass hierarchy. The lowest value of $\sin ^{2} 2 \theta_{13}$ (true) which allows us to rule out at $3 \sigma$ a $\mathrm{CP}$ conserving theory (both $0^{\circ}$ and $180^{\circ}$ ) when $\mathrm{CP}$ is maximally violated in Nature, is plotted in the figure as the sensitivity reach for maximal $\mathrm{CP}$ violation.

The close similarity of the sensitivity reaches displayed in the two panels of Fig. 2 is in line with expectation ${ }^{7}$. From the approximate symmetry

$$
P_{e \mu} \longleftrightarrow P_{\bar{e} \bar{\mu}} \text { under } \delta_{\mathrm{CP}} \longleftrightarrow-\delta_{\mathrm{CP}}
$$

noted earlier from Eq. (1), and recalling that the data involves $\nu$ as well as $\bar{\nu}$ beams, it is to be expected that results for $\delta_{\mathrm{CP}}$ (true) $=90^{\circ}$ and $\delta_{\mathrm{CP}}$ (true) $=270^{\circ}$ will be almost identical ${ }^{8}$. More explicitly, the statistics for $\delta_{\mathrm{CP}}$ (true) $=270^{\circ}$ is comparatively poor for neutrinos with $\mathrm{NH}$ true, which is made up by the higher statistics for antineutrinos. The effect of the relatively lower statistics for neutrinos shows up mainly as additional degeneracies. As discussed in the previous section, the better energy resolution and lower threshold of the TASD results in an amelioration of the correlation and degeneracies and the islands appearing in Fig. 5 of [41] are absent here.

We have assumed NH to be the true mass hierarchy in these plots. This is not expected to be a serious issue as at this baseline matter effects are small. In fact, the symmetry in Eq. (6) becomes exact if in addition to $\delta_{\mathrm{CP}} \leftrightarrow-\delta_{\mathrm{CP}}$ the neutrino mass hierarchy is also flipped. In view of this, for $\mathrm{IH}$ true, the left (right) panel of Fig. 2 would match with $\delta_{\mathrm{CP}}($ true $)=270^{\circ}\left(\delta_{\mathrm{CP}}(\right.$ true $\left.)=90^{\circ}\right)$. Of course, this argument rests on Eq. (1), which is an approximation, while the results are based on the exact expressions. We have checked that indeed taking IH to be true gives very similar results.

It is seen from Fig. 2 that in both panels the sensitivity reach for maximal $\mathrm{CP}$ violation improves sharply with $\gamma$ initially, and then it more or less saturates beyond $\gamma \gtrsim 500$. This can be

\footnotetext{
${ }^{7}$ This feature was also noted in [41] where the detector was a 50 kton magnetized iron calorimeter.

${ }^{8}$ This symmetrical behavior breaks down for larger values of $\sin ^{2} 2 \theta_{13}$ (true). See later.
} 

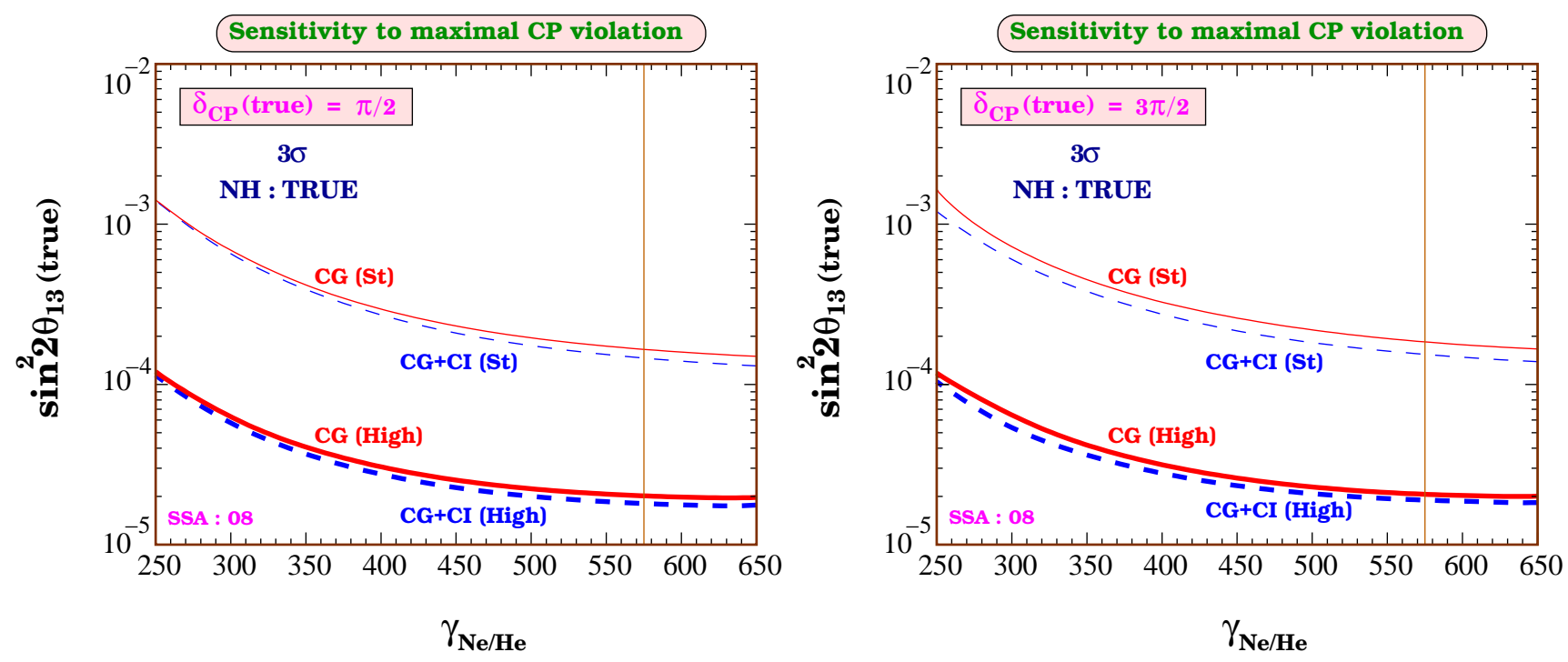

Figure 2: The $3 \sigma \sin ^{2} 2 \theta_{13}$ (true) reach for sensitivity to "maximal $\mathrm{CP}$ violation". The red solid curves (marked as 'CG') are for the CERN-TASD@LNGS alone while the blue dashed lines (marked as 'CG+CI') are for the combined data from CERN-TASD@LNGS and CERNICAL@INO. The results are shown as a function of the Lorentz boost for ${ }^{18} \mathrm{Ne}$ and ${ }^{6} \mathrm{He}$ (taken same for both ions), for $\delta_{\mathrm{CP}}$ (true) $=90^{\circ}$ (left panel) and $\delta_{\mathrm{CP}}$ (true) $=270^{\circ}$ (right panel). Thick lines (marked "High") are for $5 \times\left(1.1 \times 10^{19}\right)$ useful ${ }^{18} \mathrm{Ne}$ and ${ }^{8} \mathrm{~B}$ decays and $5 \times\left(2.9 \times 10^{19}\right)$ useful ${ }^{6} \mathrm{He}$ and ${ }^{8} \mathrm{Li}$ decays, while thin lines (marked "St") are for $5 \times\left(1.1 \times 10^{18}\right)$ and $5 \times\left(2.9 \times 10^{18}\right)$ useful ion decays respectively.

understood as follows. For $L=730 \mathrm{~km}, P_{e \mu}$ peaks around $1.5 \mathrm{GeV}$, and is very small beyond $\sim 3$ $\mathrm{GeV}$. For ${ }^{18} \mathrm{Ne}$ and ${ }^{6} \mathrm{He}$ ions, the peak (anti)neutrino energy reaches $1.5 \mathrm{GeV}$ for $\gamma \simeq 430$. For $\gamma>430$, while the peak energy shifts linearly to higher values and the total flux increases as $\gamma^{2}$, the flux at the energy $E \simeq 1.5$ where $P_{e \mu}$ is high remains roughly the same (cf. Fig. 1 of [41]). Therefore, for all $\gamma>430$ we do not see any marked improvement.

In Fig. 2 results are shown for the standard luminosity as well as for the case where the total statistics is increased by a factor of ten. Increasing the luminosity has a significant impact on the sensitivity of the experiment. From the figure one can read off that for $\gamma=575$ and $\delta_{\mathrm{CP}}($ true $)=90^{\circ}$, CP violation can be discovered at $3 \sigma$ for $\sin ^{2} 2 \theta_{13}$ (true) $>1.6 \times 10^{-4}$ for the standard luminosity and for $\sin ^{2} 2 \theta_{13}$ (true) $>1.97 \times 10^{-5}$ if luminosity is increased by a factor of ten. For $\delta_{\mathrm{CP}}($ true $)=270^{\circ}$, these limits are only slightly worse, namely, $\sin ^{2} 2 \theta_{13}$ (true) $>1.8 \times 10^{-4}$ and $\sin ^{2} 2 \theta_{13}$ (true) $>2.03 \times 10^{-5}$, respectively. Note that the results are for the NH true case but, as discussed earlier, for IH true the numbers will hardly change.

In Fig. 3 we show the impact of changing the detector characteristics on the maximal CP violation sensitivity of the TASD@LNGS set-up. The sensitivity is shown in this figure as a function of $\gamma$ for a 5 -year run with the reference choices of the $(\nu, \bar{\nu})$ luminosities. The left panel shows the effect of changing the background fraction from the standard assumed value of $10^{-3}$ (red solid lines). We have shown for comparison results for background fractions of $10^{-2}$ (black dashed 

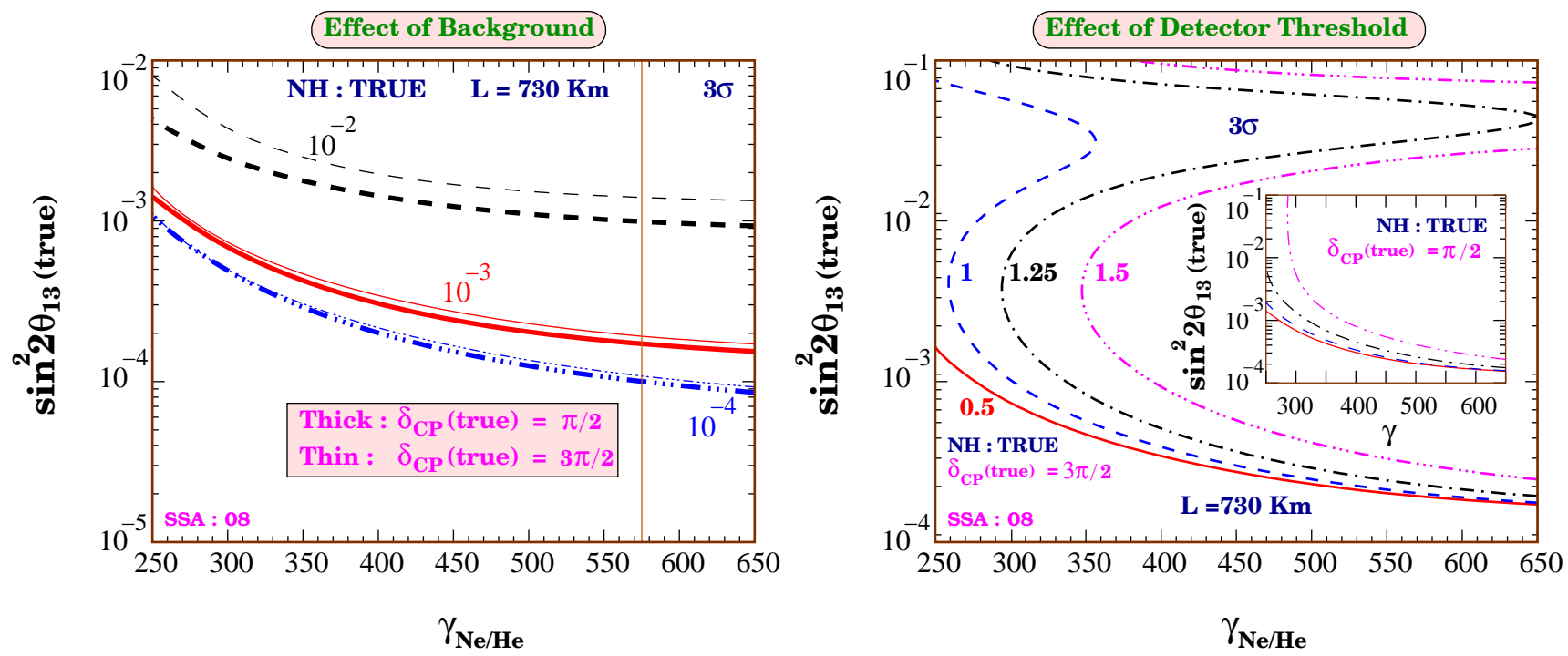

Figure 3: Effect of changing the detector characteristics on the "maximal CP violation" sensitivity. The left panel shows the impact of changing the background fraction to one-tenth or ten times the reference value of $10^{-3}$ (red solid curves). Results are shown for $\delta_{\mathrm{CP}}$ (true) $=90^{\circ}$ by thick lines and $\delta_{\mathrm{CP}}$ (true) $=270^{\circ}$ by thin lines. The location of $\gamma=575$ is also shown. The right panel shows the effect on changing the detector threshold. Results for $\delta_{\mathrm{CP}}$ (true) $=90^{\circ}$ are shown in the inset. The red solid curve corresponds to a threshold of $500 \mathrm{MeV}$ while the blue dashed, black-dot-dashed, and pink triple-dot-dashed curves correspond to thresholds of $1 \mathrm{GeV}, 1.25 \mathrm{GeV}$, and $1.5 \mathrm{GeV}$, respectively.

lines) and $10^{-4}$ (blue triple-dotted-dashed lines). We conclude (at $\gamma=575$ ) that while lowering the background fraction by a factor of 10 improves the sensitivity by a factor of 1.7, a 10-fold increase in the background would deteriorate the sensitivity by a factor of 6 . Right panel of the figure shows the impact of changing the detector threshold on the CP sensitivity. Increasing the threshold could help us in reducing the backgrounds ${ }^{9}$. We see that for $\delta_{\mathrm{CP}}$ (true) $=270^{\circ}$ increasing the detector threshold results in the appearance of degenerate solutions. For $\delta_{\mathrm{CP}}$ (true) $=90^{\circ}$ (inset) the effect is less severe; however, the sensitivity falls for both choices of $\delta_{\mathrm{CP}}$ (true) as the threshold is increased. The appearance of clone solutions (multiple solutions for the same $\gamma$ at different values of $\sin ^{2} 2 \theta_{13}$ (true)) when the threshold is increased beyond $1 \mathrm{GeV}$ reflects the important role of lower energy (i.e., lower oscillation wavelength) neutrinos in this analysis. A strength of the scintillator detector is the scope of inclusion of the low energy data by virtue of the permissible lower threshold. We have also studied the effect of changing the bin size of the data on the CP sensitivity of our experimental set-up. We find the bin size as large as $1 \mathrm{GeV}$ could also be accommodated without significantly deteriorating the $\mathrm{CP}$ violation sensitivity of the experiment.

Fig. 2 gives the "maximal $\mathrm{CP}$ violation" sensitivity reach, i.e., the ability to distinguish $\delta_{\mathrm{CP}}($ true $)=90^{\circ}$ or $270^{\circ}$ from no $\mathrm{CP}$ violation using the CERN-TASD@LNGS detector. These

\footnotetext{
${ }^{9}$ The atmospheric neutrino background would be larger at lower energies. Even backgrounds from neutral current events could be reduced by going to higher threshold.
} 


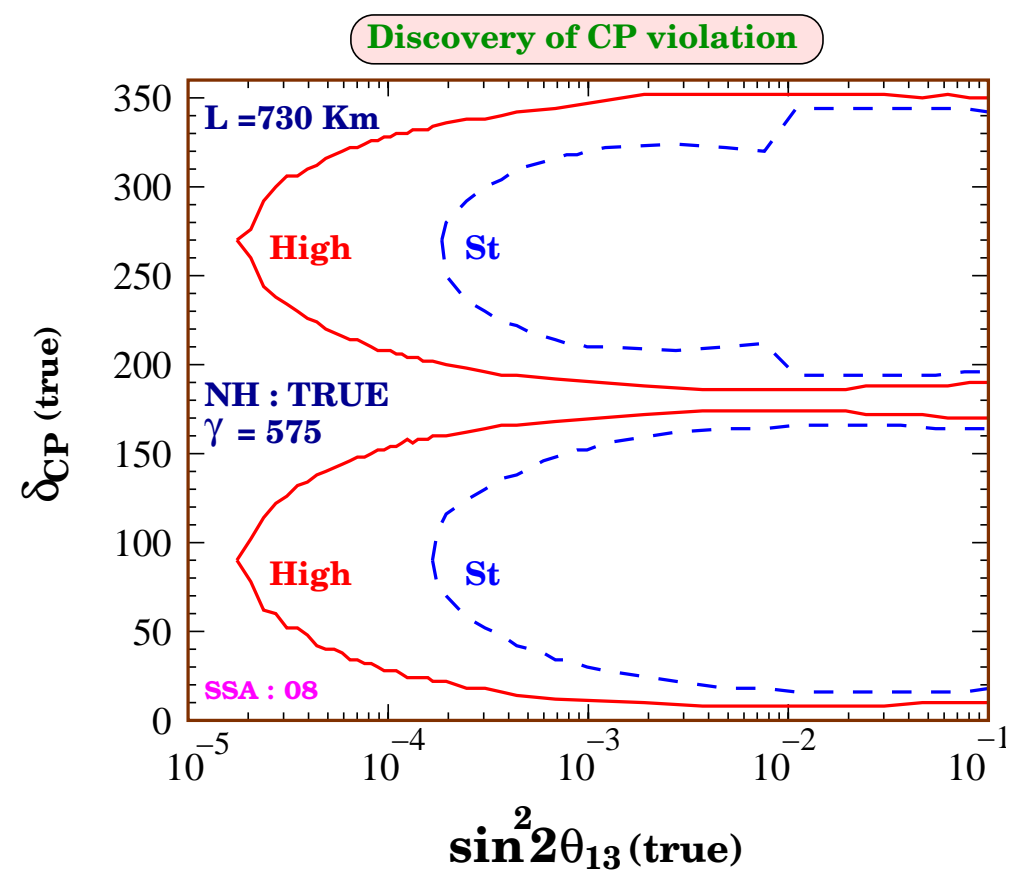

Figure 4: The area enclosed by the curves represents the $3 \sigma$ range of $\delta_{\mathrm{CP}}$ (true) as a function of $\sin ^{2} 2 \theta_{13}$ (true) for which the data can be used to rule out the CP-conserving scenario using the CERN-Gran Sasso reference TASD set-up with ${ }^{18} \mathrm{Ne}$ and ${ }^{6} \mathrm{He}$ as source ions. The Lorentz boost (the same for both ions) is fixed at 575. Results are shown for 5 years run with the reference luminosity (blue dashed curves) as well as for a luminosity which is one order higher (red solid curves).

choices of $\delta_{\mathrm{CP}}$ (true) are the extremal ones. What about other values of $\delta_{\mathrm{CP}}$ (true)? In Fig. 4 we show for $\gamma=575$, the range of $\delta_{\mathrm{CP}}$ (true) as a function of $\sin ^{2} 2 \theta_{13}$ (true) for which one can use the data to rule out no $\mathrm{CP}$ violation at the $3 \sigma$ level. We show results with $5 \times\left(1.1 \times 10^{18}\right)$ and $5 \times\left(2.9 \times 10^{18}\right)$ useful ion decays in neutrino and antineutrino modes respectively (the blue dashed curves) as well as those with one order of magnitude enhanced number of events in both channels (red solid curves). At the sensitivity reach values of $\sin ^{2} 2 \theta_{13}$ (true) displayed in Fig. 2, this range is reduced to two points. The range grows rapidly as $\sin ^{2} 2 \theta_{13}$ (true) increases to about $1 \times 10^{-3}$ beyond which it saturates, except for the kinks in the third and fourth quadrants of $\delta_{\mathrm{CP}}$ (true) around $\sin ^{2} 2 \theta_{13}$ (true) $=1 \times 10^{-2}$, which arise from clone solutions. For $\sin ^{2} 2 \theta_{13}$ (true) $\geq 10^{-3}$ we should be able to discover $\mathrm{CP}$ violation for $64 \%$ of the possible $\delta_{\mathrm{CP}}$ (true) values for the standard luminosity. If luminosity was enhanced by a factor of 10 , this would improve to $88 \%$ of the possible $\delta_{\mathrm{CP}}$ (true) values. In fact, for 10 times larger statistics, CP violation can be discovered for $64 \%$ of the possible $\delta_{\mathrm{CP}}$ (true) values for $\theta_{13}$ as low as $\sin ^{2} 2 \theta_{13}$ (true) $\geq 8 \times 10^{-5}$. 

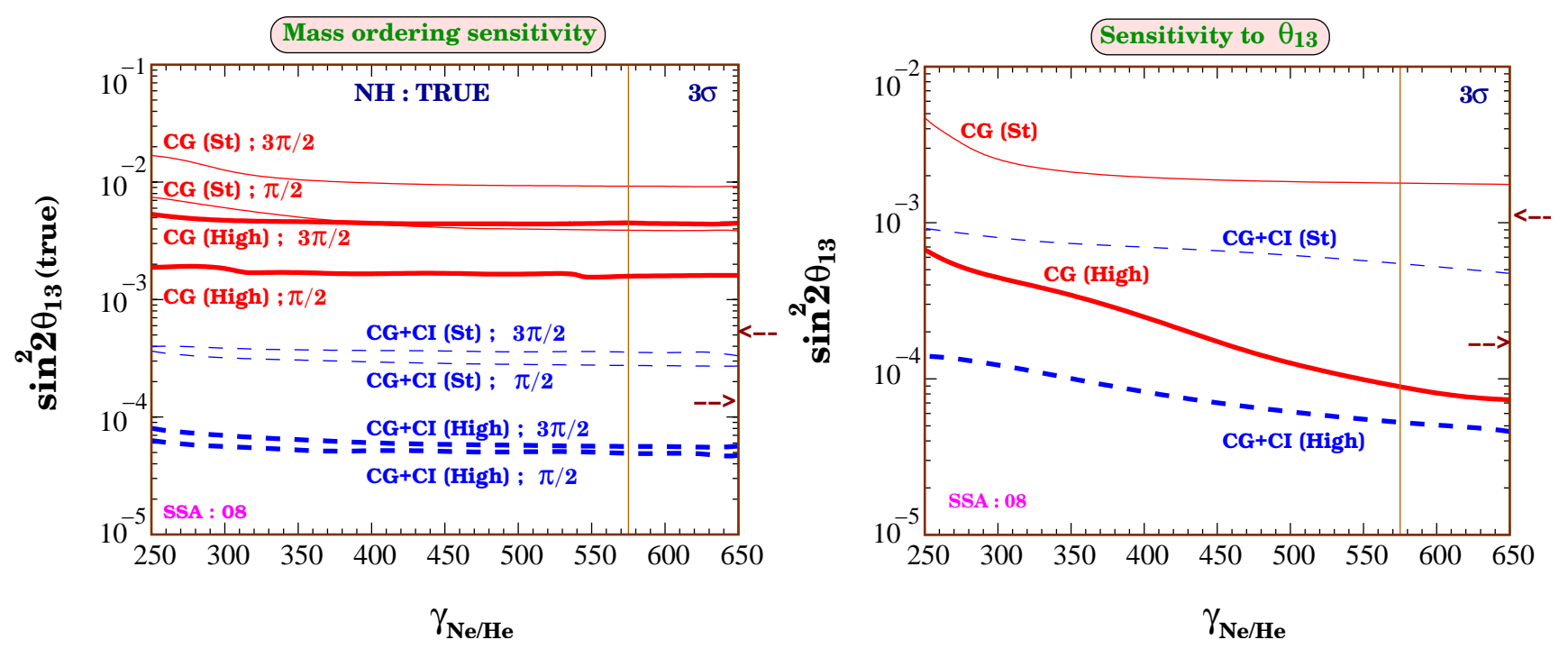

Figure 5: The left and right panels depict the $\operatorname{sgn}\left(\Delta m_{31}^{2}\right)$ sensitivity reach and the $\sin ^{2} 2 \theta_{13}$ sensitivity reach, respectively, at $3 \sigma$ as a function of the boost factor for ${ }^{18} \mathrm{Ne}$ and ${ }^{6} \mathrm{He}$. In both panels, the red solid lines are for CERN-TASD@LNGS alone while the blue dashed lines are for the combined data from CERN-ICAL@INO and CERN-TASD@LNGS. Results for $\delta_{\mathrm{CP}}$ (true) $=\pi / 2$ and $3 \pi / 2$ are shown. Thick lines are for $5 \times\left(1.1 \times 10^{19}\right)$ useful ${ }^{18} \mathrm{Ne}$ and ${ }^{8} \mathrm{~B}$ decays and $5 \times\left(2.9 \times 10^{19}\right)$ useful ${ }^{6} \mathrm{He}$ and ${ }^{8} \mathrm{Li}$ decays, while thin lines are for $5 \times\left(1.1 \times 10^{18}\right)$ and $5 \times\left(2.9 \times 10^{18}\right)$ useful ion decays respectively. The sensitivity reaches for the CERN-ICAL@INO set-up alone are indicated for both luminosities (for $\delta_{\mathrm{CP}}$ (true) $=0$ in the left panel) by arrows on the right side of the panels. The location of $\gamma=575$ is shown.

\section{Two-baseline combined results}

We are now in a position to discuss the benefits accruing from combining the results of the two detectors: one at a near-magic baseline distance and the other located $730 \mathrm{~km}$ from the source. We discuss in turn the sensitivity reaches of this combined set-up to maximal CP violation, $\sin ^{2} 2 \theta_{13}$, and the mass hierarchy.

Recall that the very long baseline detector is a magnetized iron calorimeter at the India-based Neutrino Observatory at a distance of $7152 \mathrm{~km}$ from CERN. For this detector we consider ${ }^{8} \mathrm{~B}$ and ${ }^{8} \mathrm{Li}$ as the source ions for $\nu$ and $\bar{\nu}$ beams and we assume that both are accelerated to a reference Lorentz boost $\gamma=650$. The reference choice for the number of useful ${ }^{8} \mathrm{~B}\left({ }^{8} \mathrm{Li}\right)$ ion decays is $5 \times\left(1.1 \times 10^{18}\right)\left(5 \times\left(2.9 \times 10^{18}\right)\right)$. We also show results for a 10 times enhanced luminosity.

\subsection{CP Sensitivity of the Combined Set-up}

We first probe the impact of adding the data from the CERN-ICAL@INO set-up on the sensitivity reach in $\sin ^{2} 2 \theta_{13}$ (true) for maximal CP violation. We chose to use the CERN-LNGS baseline with ${ }^{18} \mathrm{Ne}$ and ${ }^{6} \mathrm{He}$ as source ions since it was shown in [41] to be the ideal set-up for probing $\delta_{\mathrm{CP}}$. In Fig. 2 is shown (blue dashed curves) the impact of adding the CERN-ICAL@INO ${ }^{8} \mathrm{~B}$ and ${ }^{8} \mathrm{Li}$ results 
on the sensitivity to maximal $\mathrm{CP}$ violation. It is seen that there is insignificant improvement in the sensitivity. We can conclude that the CERN-LNGS baseline, which is the optimal choice as a stand-alone set-up for CP measurements, remains so after the addition of the new magic baseline data. This should not be surprising since at the magic baseline there is no $\delta_{\mathrm{CP}}$ dependence, and the $\mathrm{CP}$ violation measurement of the $730 \mathrm{~km}$ experiment is almost devoid of parameter degeneracies.

\subsection{The $\operatorname{sgn}\left(\Delta \mathrm{m}_{31}^{2}\right)$ Sensitivity Reach}

Next, we consider the potential of the two-baseline Beta-beam set-up to explore the nature of the neutrino mass hierarchy. The " $\operatorname{sgn}\left(\Delta m_{31}^{2}\right)$ sensitivity reach" is defined as the range of values of $\sin ^{2} 2 \theta_{13}$ (true) for which the experiment could rule out the wrong hierarchy at $3 \sigma$. We show this as a function of the boost of the ${ }^{18} \mathrm{Ne}$ and ${ }^{6} \mathrm{He}$ ions in the left panel of Fig. 5. The boost for the ${ }^{8} \mathrm{~B}$ and ${ }^{8} \mathrm{Li}$ source ions for CERN-ICAL@INO is fixed at $\gamma=650$. We have shown results for $\delta_{\mathrm{CP}}($ true $)=90^{\circ}$ and $270^{\circ}$ and assuming $\mathrm{NH}$ to be true. The solid lines show the $\operatorname{sgn}\left(\Delta m_{31}^{2}\right)$ sensitivity of CERN-TASD@LNGS alone while the dashed lines give the sensitivity when the two data sets are combined. The $\operatorname{sgn}\left(\Delta m_{31}^{2}\right)$ sensitivity reaches for the CERN-ICAL@INO set-up with $\gamma=650$ with the standard and enhanced luminosities (for $\delta_{\mathrm{CP}}$ (true) $=0$ ) are indicated in the figure by arrows. In Table 4 we give for a fixed $\gamma$, the sensitivity reach in $\sin ^{2} 2 \theta_{13}$ (true) for determining the mass hierarchy for the single and combined baseline set-ups considered in this paper. A comparison with the sensitivity reach envisaged for the optimized two-baseline neutrino factory from Ref. [4] is also given for comparison. From the figure and the table we note that the hierarchy sensitivity is rather poor for the CERN-TASD@LNGS set-up alone since the baseline is not large enough to capture enough matter effect and is almost independent of the value of $\gamma$. As shown in [23], the CERN-ICAL@INO experiment has a very good hierarchy sensitivity. Notice the near-independence on $\delta_{\mathrm{CP}}$ (true) - a feature of the magic baseline. For the combined two-baseline set-up we achieve an exceptional sensitivity such that (for $\delta_{\mathrm{CP}}($ true $)=90^{\circ}$ ) the mass hierarchy could be determined at $3 \sigma$ if $\sin ^{2} 2 \theta_{13}$ (true) $>2.7 \times 10^{-4}$ and $4.64 \times 10^{-5}$ for the standard and ten times enhanced luminosity respectively. All results presented are for NH true, however, we have checked that results with IH true are similar.

\subsection{The $\sin ^{2} 2 \theta_{13}$ Sensitivity Reach}

Finally, we turn to the expected sensitivity of the combined set-up to $\theta_{13}$. We define the performance indicator " $\sin ^{2} 2 \theta_{13}$ sensitivity reach" as follows. We generate the data at $\sin ^{2} 2 \theta_{13}($ true $)=$ 0 and determine the values of $\sin ^{2} 2 \theta_{13}$ that could fit this data within a chosen confidence level, allowing all oscillation parameters, including $\delta_{\mathrm{CP}}$ and the mass hierarchy, to vary freely in the fit. We also marginalize over the normalization of the PREM density profile. This is applicable when the experiment has observed no $\theta_{13}$ driven signal and at best can put an upper limit on the still allowed $\sin ^{2} 2 \theta_{13}$. The $3 \sigma$ projected $\sin ^{2} 2 \theta_{13}$ sensitivity as a function of $\gamma$ for ${ }^{18} \mathrm{Ne}$ and ${ }^{6} \mathrm{He}$ is shown in the right panel of Fig. 5. The boost of ${ }^{8} \mathrm{~B}$ and ${ }^{8} \mathrm{Li}$ for the CERN-ICAL@INO data is fixed at $\gamma=650$. The red solid lines in the figure show the $\sin ^{2} 2 \theta_{13}$ sensitivity for CERN-TASD@LNGS alone, while the dashed lines are what we expect by combining the two data sets. The sensitivity reach in $\sin ^{2} 2 \theta_{13}$ for the CERN-ICAL@INO is given in Table 4 and is indicated in the figure by an arrow. As can be seen from Table 4, the sensitivity reach for CERN-TASD@LNGS alone is 
comparable to that of CERN-ICAL@INO taken alone. For ten times enhanced statistics the corresponding $\sin ^{2} 2 \theta_{13}$ sensitivity reach of CERN-ICAL@INO and CERN-TASD@LNGS taken alone are, $\sin ^{2} 2 \theta_{13}<1.76 \times 10^{-4}$ and $\sin ^{2} 2 \theta_{13}<8.59 \times 10^{-5}$ respectively. A combination of the two data sets improves the performance tremendously. For the standard luminosity we could reach down to $\sin ^{2} 2 \theta_{13}<5.46 \times 10^{-4}$, which could be further improved to $\sin ^{2} 2 \theta_{13}<5.26 \times 10^{-5}$ in the event that ten times larger statistics becomes feasible.

\section{Discussions and Conclusions}

In previous papers $[22,23]$ we have shown that a Beta-beam experiment where $\nu_{e}$ and $\bar{\nu}_{e}$ beams produced using ${ }^{8} \mathrm{~B}$ and ${ }^{8} \mathrm{Li}$ ions respectively are sent from CERN to the magnetized iron calorimeter ICAL at INO results in tremendous sensitivity to $\theta_{13}$ and the mass hierarchy. This unprecedented sensitivity stems from a combined effect of the CERN-INO distance being magic, as well as the energy of the Beta-beam being conducive to encountering near-maximal matter effects, thereby resulting in substantial enhancement of the oscillation probability and hence statistics. However, while the baseline being magic and the beam energy being in the multi-GeV regime is a virtue for probing $\theta_{13}$ and the mass hierarchy, it becomes a serious drawback for $\mathrm{CP}$ violation studies. This necessitates the deployment of a second baseline option with a lower energy neutrino beam for investigating all the three issues simultaneously. The optimal baseline and ion source option for $\mathrm{CP}$ violation studies was shown in [41] to be $L=600-900 \mathrm{~km}$ and the ${ }^{18} \mathrm{Ne}$ and ${ }^{6} \mathrm{He}$ combination with intermediate values of $\gamma$. In this paper we studied the sensitivity reach of the combined data sets from the CERN to INO magical Beta-beam set-up and a CERN to Gran Sasso Beta-beam experiment with ${ }^{18} \mathrm{Ne}$ and ${ }^{6} \mathrm{He}$ as source ions. We reiterate that even for the Neutrino Factory experiment, at least two baselines are needed in order to optimally address all the three neutrino oscillation parameters.

For the CERN to INO set-up, the baseline is $L=7152 \mathrm{~km}$ and we used ${ }^{8} \mathrm{~B}$ and ${ }^{8} \mathrm{Li}$ as sources, with boost $\gamma=650$. The far detector was taken as ICAL, a 50 kton magnetized iron calorimeter with detection efficiency of $80 \%$, charge identification efficency of $95 \%$, energy threshold of $1 \mathrm{GeV}$, energy resolution of $0.15 E$ and neutral current background fraction of $10^{-4}$. We call this set-up CERN-ICAL@INO. For the CERN to Gran Sasso sector, the baseline is $L=730 \mathrm{~km}$ and we chose ${ }^{18} \mathrm{Ne}$ and ${ }^{6} \mathrm{He}$ as source ions. The Lorentz boosts for the ${ }^{18} \mathrm{Ne}$ and ${ }^{6} \mathrm{He}$ ions are taken to be the same and allowed to vary between 250 and 650 . Because of the lower beam energy for this case it is preferable to opt for a Totally Active Scintillator Detector here. We assume an active detector mass of 50 kton with $80 \%$ detection efficiency for muons and $20 \%$ detection efficiency for electrons, energy threshold of $0.5 \mathrm{GeV}$, energy resolution of $0.03 \sqrt{E(\mathrm{GeV})}$ for muons and $0.06 \sqrt{E(\mathrm{GeV})}$ for electrons and neutral current background fraction of $10^{-3}$. We call this set-up CERN-TASD@LNGS. We show results for "standard luminosity" where we use the abovementioned detector sizes and efficiencies and $5 \times\left(1.1 \times 10^{18}\right)$ and $5 \times\left(2.9 \times 10^{18}\right)$ useful ion decays in neutrino and antineutrino modes respectively. We also show results for a situation where the statistics is ten times larger.

We probed the physics potential of this two-detector set-up with a Beta-beam as the neutrino source. We have presented our results in terms of three performance indicators. The essential results are summarized in Table 4 . For $\delta_{\mathrm{CP}}$ sensitivity we showed the $\sin ^{2} 2 \theta_{13}$ (true) 


\begin{tabular}{|c|c|c|c|c|c|c|}
\hline \multirow[t]{2}{*}{ Set-up } & \multicolumn{2}{|c|}{$\begin{array}{c}\text { Mass Ordering }(3 \sigma) \\
\text { NH (True) }\end{array}$} & \multicolumn{2}{|c|}{$\begin{array}{c}\text { CP Sensitivity }(3 \sigma) \\
\text { NH (True) }\end{array}$} & \multicolumn{2}{|c|}{$\sin ^{2} 2 \theta_{13}$ Sensitivity $(3 \sigma)$} \\
\hline & $\begin{array}{c}1.1 \times 10^{18} \\
\& \\
2.9 \times 10^{18}\end{array}$ & $\begin{array}{c}1.1 \times 10^{19} \\
\& \\
2.9 \times 10^{19}\end{array}$ & $\begin{array}{c}1.1 \times 10^{18} \\
\& \\
2.9 \times 10^{18}\end{array}$ & $\begin{array}{c}1.1 \times 10^{19} \\
\& \\
2.9 \times 10^{19}\end{array}$ & $\begin{array}{c}1.1 \times 10^{18} \\
\& \\
2.9 \times 10^{18}\end{array}$ & $\begin{array}{c}1.1 \times 10^{19} \\
\& \\
2.9 \times 10^{19}\end{array}$ \\
\hline $\begin{array}{c}\text { CERN-INO } \\
\gamma=650,7152 \mathrm{Km}\end{array}$ & $\begin{array}{c}4.7 \times 10^{-4} \\
\left(4.9 \times 10^{-4}\right) \\
\end{array}$ & $\begin{array}{c}9.4 \times 10^{-5} \\
\left(1.2 \times 10^{-4}\right) \\
\end{array}$ & $\begin{array}{c}\text { Not } \\
\text { possible }\end{array}$ & $\begin{array}{c}\text { Not } \\
\text { possible }\end{array}$ & $1.14 \times 10^{-3}$ & $1.76 \times 10^{-4}$ \\
\hline $\begin{array}{c}\text { CERN-LNGS } \\
\gamma=575,730 \mathrm{Km}\end{array}$ & $\begin{array}{c}3.89 \times 10^{-3} \\
\left(9.23 \times 10^{-3}\right)\end{array}$ & $\begin{array}{c}1.58 \times 10^{-3} \\
\left(4.48 \times 10^{-3}\right)\end{array}$ & $\begin{array}{c}1.6 \times 10^{-4} \\
\left(1.8 \times 10^{-4}\right)\end{array}$ & $\begin{array}{c}1.97 \times 10^{-5} \\
\left(2.03 \times 10^{-5}\right)\end{array}$ & $1.78 \times 10^{-3}$ & $8.59 \times 10^{-5}$ \\
\hline $\begin{array}{l}\text { CERN-BOULBY } \\
\gamma=575,1050 \mathrm{Km}\end{array}$ & $\begin{array}{c}2.49 \times 10^{-3} \\
\left(7.87 \times 10^{-3}\right)\end{array}$ & $\begin{array}{l}2.19 \times 10^{-4} \\
\left(4.1 \times 10^{-3}\right)\end{array}$ & $\begin{array}{c}1.85 \times 10^{-4} \\
\left(2.02 \times 10^{-4}\right)\end{array}$ & $\begin{array}{c}1.99 \times 10^{-5} \\
\left(2.04 \times 10^{-5}\right)\end{array}$ & $1.41 \times 10^{-3}$ & $1.45 \times 10^{-4}$ \\
\hline $\begin{array}{c}\text { CERN-LNGS } \\
\gamma=575,730 \mathrm{Km} \\
+ \\
\text { CERN-INO } \\
\gamma=650,7152 \mathrm{Km}\end{array}$ & $\begin{array}{c}2.7 \times 10^{-4} \\
\left(3.58 \times 10^{-4}\right)\end{array}$ & $\begin{array}{l}4.64 \times 10^{-5} \\
\left(5.45 \times 10^{-5}\right)\end{array}$ & $\begin{array}{c}1.42 \times 10^{-4} \\
\left(1.49 \times 10^{-4}\right)\end{array}$ & $\begin{array}{c}1.78 \times 10^{-5} \\
\left(1.88 \times 10^{-5}\right)\end{array}$ & $5.46 \times 10^{-4}$ & $5.26 \times 10^{-5}$ \\
\hline $\begin{array}{c}\text { CERN-BOULBY } \\
\gamma=575,1050 \mathrm{Km} \\
+ \\
\text { CERN-INO } \\
\gamma=650,7152 \mathrm{Km} \\
\end{array}$ & $\begin{array}{c}2.67 \times 10^{-4} \\
\left(3.37 \times 10^{-4}\right)\end{array}$ & $\begin{array}{c}4.57 \times 10^{-5} \\
\left(5.17 \times 10^{-5}\right)\end{array}$ & $\begin{array}{c}1.63 \times 10^{-4} \\
\left(1.76 \times 10^{-4}\right)\end{array}$ & $\begin{array}{c}1.8 \times 10^{-5} \\
\left(1.87 \times 10^{-5}\right)\end{array}$ & $6.1 \times 10^{-4}$ & $6.69 \times 10^{-5}$ \\
\hline \multicolumn{7}{|c|}{$\begin{array}{l}\text { Optimized Neutrino Factory set-up with two improved golden detectors (50 kton each) placed at } \\
L=4000 \mathrm{~km} \& 7500 \mathrm{~km} \text { respectively. } E_{\mu}=20 \mathrm{GeV} \& \text { total } 5 \times 10^{21} \text { decays for } \mu^{-} \& \mu^{+} \text {each. }\end{array}$} \\
\hline $\begin{array}{c}\text { Optimized } \\
\text { Neutrino Factory }\end{array}$ & $\begin{array}{r}4.5> \\
\left(100 \% \text { of } \delta_{\mathrm{CP}}\right. \\
\end{array}$ & $\begin{array}{l}10^{-5} \\
\text { true) coverage) }\end{array}$ & 1.5 & $10^{-5}$ & & $10^{-5}$ \\
\hline
\end{tabular}

Table 4: Performances of various Beta-beam set-ups at $3 \sigma$ in addressing the key unsolved issues: mass ordering, CP violation and $\sin ^{2} 2 \theta_{13}$ sensitivity reach. For CP sensitivity and mass ordering, the minimum values of $\sin ^{2} 2 \theta_{13}$ (true) required for a positive conclusion are presented. Results are shown for a five-year run with the reference luminosity: $1.1 \times 10^{18}\left(2.9 \times 10^{18}\right)$ useful ion decays per year in the $\nu(\bar{\nu})$ mode as well as one order of magnitude higher statistics. The numbers without (with) parantheses correspond to $\delta_{\mathrm{CP}}($ true $)=90^{\circ}\left(\delta_{\mathrm{CP}}\right.$ (true) $\left.=270^{\circ}\right)$. Note that the $\sin ^{2} 2 \theta_{13}$ sensitivity reach is independent of the value of $\delta_{\mathrm{CP}}$ (true) and the true mass ordering because the prospective "data" have been generated at $\theta_{13}=0$. The CERN-INO baseline is insensitive to $\delta_{\mathrm{CP}}$. For comparison, the expectations from an optimized two-baseline Neutrino Factory set-up with upgraded magnetized iron detectors are also listed [4, 40]. 
reach of the experiment to distinguish maximal $\mathrm{CP}$ violation from a $\mathrm{CP}$-conserving scenario. We displayed the results for the CERN-TASD@LNGS experiment by itself and when data from here are combined with that from CERN-ICAL@INO. From CERN-TASD@LNGS alone we obtain magnificent sensitivity to $\delta_{\mathrm{CP}}$. For $\mathrm{NH}$ true and $\gamma=575$ for ${ }^{18} \mathrm{Ne}$ and ${ }^{6} \mathrm{He}$ and with the standard luminosity, maximal CP violation can be established at $3 \sigma$ at CERN-TASD@LNGS if $\sin ^{2} 2 \theta_{13}$ (true) $>1.6 \times 10^{-4}$ for $\delta_{\mathrm{CP}}($ true $)=90^{\circ}$. With ten times more statistics this limit gets pushed to $\sin ^{2} 2 \theta_{13}$ (true) $>1.97 \times 10^{-5}$. Addition of the CERN-ICAL@INO data does not improve these limits significantly. We also explored the dependence of maximal CP violation sensitivity of CERN-TASD@LNGS to the detector characteristics of TASD. Results are similar if IH is true.

Measurement of $\theta_{13}$ and mass hierarchy can be performed extremely well at CERN-ICAL@INO. We studied the impact of adding the data from CERN-TASD@LNGS on the final combined sensitivity to these parameters. For the mixing angle $\theta_{13}$ we defined the $\sin ^{2} 2 \theta_{13}$ sensitivity as the range of $\sin ^{2} 2 \theta_{13}$ which could fit at $3 \sigma$ the data generated for $\sin ^{2} 2 \theta_{13}$ (true) $=0$, after marginalization over all oscillation parameters and the density profile. We found that the $\sin ^{2} 2 \theta_{13}$ sensitivity of both set-ups taken alone are comparable. CERN-ICAL@INO could limit $\sin ^{2} 2 \theta_{13}<$ $1.14 \times 10^{-3}$ for the standard luminosity and with $\gamma=650$, while CERN-TASD $@$ LNGS could restrict $\sin ^{2} 2 \theta_{13}<1.78 \times 10^{-3}$ with $\gamma=575$. For the enhanced luminosity these limits would be $\sin ^{2} 2 \theta_{13}<1.76 \times 10^{-4}$ and $\sin ^{2} 2 \theta_{13}<8.59 \times 10^{-5}$ respectively. However, when added together, the combined data can limit $\sin ^{2} 2 \theta_{13}<5.46 \times 10^{-4}$ for the standard luminosity and $\sin ^{2} 2 \theta_{13}<$ $5.26 \times 10^{-5}$ with a factor of ten enhanced luminosity.

The sensitivity to $\operatorname{sgn}\left(\Delta m_{31}^{2}\right)$ is defined in terms of the $\sin ^{2} 2 \theta_{13}$ (true) required in order to rule out the wrong hierarchy at $3 \sigma$. We had shown earlier that for CERN-ICAL@INO with $\gamma=650$ and standard luminosity the wrong inverted hierarchy can be disfavored at $3 \sigma$ if $\sin ^{2} 2 \theta_{13}$ (true) $>$ $4.7 \times 10^{-4}$ for $\delta_{\mathrm{CP}}($ true $)=90^{\circ}$. With ten times more statistics this improves to $\sin ^{2} 2 \theta_{13}($ true $)>$ $9.4 \times 10^{-5}$. We found in this paper that the $\operatorname{sgn}\left(\Delta m_{31}^{2}\right)$ sensitivity of CERN-TASD@LNGS alone is rather poor in comparison. However, when we add the two data sets, we find an enhancement in the sensitivity. With data from both set-ups taken together the wrong inverted hierarchy can be disfavored at $3 \sigma$ if $\sin ^{2} 2 \theta_{13}$ (true) $>2.7 \times 10^{-4}$ with the standard luminosity when $\delta_{\mathrm{CP}}$ (true) $=90^{\circ}$. With ten times more statistics it would become possible if $\sin ^{2} 2 \theta_{13}$ (true) $>4.64 \times 10^{-5}$. Results for $\delta_{\mathrm{CP}}$ (true) $=270^{\circ}$ are similar, and so are the sensitivity reaches for $\mathrm{IH}$ true.

Another choice for an intermediate baseline could be sending a beam from CERN to the Boulby mine in UK. The CERN to Boulby distance is about $1050 \mathrm{~km}$. Since INO and Boulby mine are in opposite hemispheres with respect to CERN, it might be easier to contemplate a decay ring design which could be used to send beams to both these (plausible) detector sites. While in the text we explicitly presented and discussed results for the CERN-LNGS baseline, Table 4 shows results for the CERN-Boulby mine set-up as well. All detector characteristics are taken to be the same as for the LNGS case. The sensitivity reach can be quantitatively seen to be comparable for both the intermediate baseline options when combined with CERN-INO data, with the CERN-LNGS baseline being marginally better.

The sensitivity obtained in the set-ups considered here could be compared to that obtained in a benchmark high $\gamma$ set-up considered in [33]. In this paper, the authors proposed using a $\gamma=350{ }^{18} \mathrm{Ne}$ and ${ }^{6} \mathrm{He}$ Beta-beam sent to a megaton water detector located at a distance of 730 $\mathrm{km}$ from CERN. For total exposure of 5.0 Mton-year, this set-up returns a $3 \sigma \sin ^{2} 2 \theta_{13}$ sensitivity of $5.7 \times 10^{-4}$. Normal mass hierarchy discovery reach at $3 \sigma$ ranges between $2.4 \times 10^{-3}$ and 
$1.6 \times 10^{-2}$ depending on the choice of $\delta_{\mathrm{CP}}$ (true). Maximal $\mathrm{CP}$ violation can be established at $3 \sigma$ if $\sin ^{2} 2 \theta_{13}$ (true) $\geq 5.2 \times 10^{-5}$ for $\delta_{\mathrm{CP}}($ true $)=\pi / 2$ and $\sin ^{2} 2 \theta_{13}$ (true) $\geq 5.5 \times 10^{-5}$ for $\delta_{\mathrm{CP}}($ true $)=3 \pi / 2$.

The Beta-beam set-ups discussed here have a reach comparable to those obtained from experiments based on optimised Neutrino Factories, see Table 4. Here, the reference Neutrino Factory set-up we have considered is the version with two "golden magnetized detectors" of 50 kton each at $L=4000 \mathrm{~km}$ and $7500 \mathrm{~km}$, a detector threshold of $1 \mathrm{GeV}, 5$ years $\mu^{+} / \mu^{-}$data with $E_{\mu}=$ $20 \mathrm{GeV}$ and a luminosity of $10^{21}$ decays per year for each polarity, with a background fraction of $5 \times 10^{-6}$. Further details of these set-ups and their resolutions and efficiencies can be found in $[4,40]$.

It is of interest to compare the sensitivity reach of our two-detector Beta-beam set-up with that chosen in [31], where the authors used $L=2000 \mathrm{~km}$ for the shorter baseline magnetized iron calorimeter detector in addition to another at the magic baseline, and ${ }^{8} \mathrm{~B}$ and ${ }^{8} \mathrm{Li}$ ions as sources for both. While they use a smaller boost factor of 350 for the beam, they require larger number of ion decays than we have used for our standard luminosity. Our results with standard luminosity are comparable to those of [31] with larger luminosity, as noted in the Introduction. Similar reach for $\mathrm{CP}$ sensitivity in the two proposals even though with different source ions and intermediate baseline is not surprising. The relative optimization between $\gamma$ and luminosity for the two sets of sources was studied in detail in [41]. It was shown that to obtain the same "physics" with ${ }^{8} \mathrm{~B} /{ }^{8} \mathrm{Li}$ and ${ }^{18} \mathrm{Ne} /{ }^{6} \mathrm{He}$, one needs 12 times larger luminosity for the former and 3.5 times larger $\gamma$ for the latter. While we have used a factor of 1.86 higher boost than that in [31], the authors of [31] consider 7.39 (2.80) times higher luminosity for their neutrino (antineutrino) mode. For lower number of ion decays the sensitivity of the set-up proposed in [31] becomes worse than our set-up. The main strength of the set-up proposed here emerges from the fact that use has been made of the optimal choice for the source ions and baselines [41] for CP, $\operatorname{sgn}\left(\Delta m_{31}^{2}\right)$ and $\theta_{13}$. The boost factors that we use, though larger, should be plausible at CERN and were optimized for the CERN-INO set-up for determining $\operatorname{sgn}\left(\Delta m_{31}^{2}\right)$ and $\theta_{13}$ [23]. For the corresponding boost possible for ${ }^{18} \mathrm{Ne}$ and ${ }^{6} \mathrm{He}$ we achieve good sensitivity to $\mathrm{CP}$ violation in the CERN-LNGS set-up. The combined data set from both detectors provide exceptional sensitivity to all three neutrino parameters. With ten times larger luminosities, which are also considered to be plausible [43], the sensitivity of our set-up escalates to unprecedented levels and are similar to those possible with a high performance two-detector Neutrino Factory set-up, using improved detectors with very low backgrounds and low threshold (see Table 4).

In conclusion, we show that a Beta-beam experiment with two carefully chosen detectors at optimal distances can provide unprecedented sensitivity to the establishment of CP-violation in the lepton sector, addressing the issue of neutrino mass ordering, and determination of the mixing angle $\theta_{13}$. We have considered a Totally Active Scintillator Detector at the shorter baseline of 730 $\mathrm{km}$ and a magnetized iron calorimeter at a near-magic baseline distance of $7152 \mathrm{~km}$, both of mass 50 kton. In tandem, they provide an exceptional sensitivity to the neutrino parameters and match the precision achievable in high performance Neutrino Factory set-ups.

\section{Acknowledgments}

The authors acknowledge the HRI cluster facilities for computation. This work has been supported 
under the XI Plan Neutrino Project at the Harish-Chandra Research Institute.

\section{A Appendix: Background Rejection}

In this Appendix we discuss some technical issues pertaining to rejection of the beam related and atmospheric neutrino backgrounds.

\section{A.1 Beam related neutral current backgrounds}

From Fig. 1, one can see that at the CERN-LNGS baseline $(L=730 \mathrm{Km})$, the expected event rate sharply depends on the choice of $\delta_{\mathrm{CP}}$ and $\theta_{13}$. Assuming $1.1 \times 10^{18}$ useful decays per year for ${ }^{18} \mathrm{Ne}$ and at $\sin ^{2} 2 \theta_{13}=10^{-3}$ with a boost factor of 575 and normal hierarchy, the variation in the signal in 5 years with $\delta_{\mathrm{CP}}$ has been depicted in Table 5 . The beam related neutral current background in this case (after folding with $10^{-3}$ suppression factor for TASD) comes out to be 23 events. So in this case, the background over signal ratio may vary over a wide range from 0.26 to 1.92. This case is quite different to that encountered in the CERN-INO Beta-beam experiment $[22,23]$ where we hardly expect any beam related background. Though the total signal to neutral current background ratio is high, we stress that an aspect which is extremely crucial is the different spectral shape of the signal compared to the background. In our analysis we have cautiously taken into account the beam related background as described in the appendix of [23].

\begin{tabular}{||c||c||c||c||c||}
\hline \hline$\delta_{\mathrm{CP}}$ & 0 & $90^{\circ}$ & $180^{\circ}$ & $270^{\circ}$ \\
\hline Signal $(5 \mathrm{yrs})$ & 67 & 90 & 35 & 12 \\
\hline \hline
\end{tabular}

Table 5: Number of $\nu$ signal events in 5 years for various $\delta_{\mathrm{CP}}$ with a 50 kton TASD detector $\left(\sin ^{2} 2 \theta_{13}=10^{-3}\right.$ and $\mathrm{NH}$ with $\left.\gamma=575\right)$.

\section{A.2 Atmospheric backgrounds}

Next we turn to the backgrounds due to atmospheric neutrinos. In the CERN-LNGS set-up, we have considered a TASD detector with an energy threshold of $500 \mathrm{MeV}$. With ${ }^{18} \mathrm{Ne}$ and $\gamma=575$, we have an energy spectrum extending upto $3.9 \mathrm{GeV}$ with a peak at $2.25 \mathrm{GeV}$. In this energy range the number of muons from atmospheric neutrinos giving tracks inside the detector along the direction of the $\beta$-beam flux are expected to be very large (see Table 6 ). Without demur one can see that the atmospheric backgrounds are huge compared to the signal rates. Also the TASD detector does not have any charge identification (CID) capability and it makes the case more arduous in the sense that we have to consider both $\mu^{-}$and $\mu^{+}$atmospheric events simultaneously as the backgrounds when we expect a signal from the neutrino beam alone. In such a situation, the timing information of the ion bunches inside the storage ring turns out to be a valuable tool to tackle these backgrounds. 


\begin{tabular}{||c||c||c||}
\hline \hline Energy range $(\mathrm{GeV})$ & $\mu^{-}$events & $\mu^{+}$events \\
\hline $0.5-4$ & 1342 & 447 \\
\hline $1-4$ & 852 & 344 \\
\hline \hline
\end{tabular}

Table 6: Expected atmospheric neutrino events along the beam direction in 5 years with a 50 kton TASD detector.

\section{A.3 Bunch size and background reduction}

For a $5 \mathrm{~T}$ magnetic field and $\gamma=575$ for ${ }^{18} \mathrm{Ne}$ ions, the radius of the curved section would be $R \sim 720 \mathrm{~m}$. The useful decays are those which occur in one of the straight sections where emitted neutrinos fly towards the direction of the detector. If $L_{r}$ is the length of one of the straight sections of the decay ring and if we demand that $L_{r} /\left(2 \pi R+2 L_{r}\right) \sim 36 \%$ (useful fraction of ion decays) then $L_{r}$ comes out to be $5818 \mathrm{~m}$. With this design, the total length of the storage ring $\left(2 \pi R+2 L_{r}\right)$ would be $16161 \mathrm{~m}$. Now if we can tolerate the effective atmospheric background over beam signal ratio to upto $10^{-1}$ then

$$
10^{-1}=\frac{\text { Atmospheric Background } \times \text { Suppression Factor }\left(S_{f}\right)}{\text { Beam Signal }}
$$

The total atmospheric background in the energy range of 0.5 to $4 \mathrm{GeV}$ is 1789 (see Table 6) and the expected lowest signal rate in 5 years from the ${ }^{18} \mathrm{Ne} \beta$-beam is 12 (with $\sin ^{2} 2 \theta_{13}=10^{-3}$ and $\delta_{\mathrm{CP}}=270^{\circ}$ ). So from Eq. A-1, we have $S_{f}=6.7 \times 10^{-4}$. To achieve this $S_{f}$, one has to ensure an excellent timing information for the signal correlating with the source bunches. This requirement puts a constraint on the maximum allowed time-length of the ion bunch $T_{b}$ inside the storage ring. The $S_{f}$ and $T_{b}$ are related in the following fashion,

$$
S_{f}=\frac{v \times T_{b} \times N_{b}}{2 \pi R+2 L_{r}} \times 0.36
$$

where $v \simeq c$ is the velocity of the ion and $N_{b}$ is the maximum number of bunches of the ion circulating inside the storage ring at the same time. One can readily see from Eq. A-2 that $S_{f}=6.7 \times 10^{-4}$ can be achieved with $12.5 \mathrm{~ns}$ time-length of the ion bunch taking $N_{b}=8$.

If we increase the threshold energy of the TASD detector from 0.5 to $1 \mathrm{GeV}$ then we can suppress the atmospheric events by a factor of 1.5 (see Table 6) and now the total atmospheric background would be 1196. But in this process, the signal rate also gets reduced from 12 to 8 , keeping the $S_{f}$ almost same.

Another important point one should keep in mind is that if we can work with one order higher luminosity of the Beta-beam flux then the signal rate will be enhanced by a factor of 10 and therefore one can relax the time length of the ion bunch from 12.5 ns to 125 ns provided that $N_{b}$ is kept fixed.

\section{References}

[1] Y. Itow et al., arXiv:hep-ex/0106019. 
[2] D. S. Ayres et al. [NOvA Collaboration], arXiv:hep-ex/0503053.

[3] http://www.hep.ph.ic.ac.uk/iss/

[4] A. Bandyopadhyay et al. [ISS Physics Working Group], arXiv:0710.4947 [hep-ph].

[5] A. Cervera, A. Donini, M. B. Gavela, J. J. Gómez-Cadenas, P. Hernandez, O. Mena and S. Rigolin, Nucl. Phys. B 579, 17 (2000) [Erratum-ibid. B 593, 731 (2001)].

[6] J. Burguet-Castell, M. B. Gavela, J. J. Gómez-Cadenas, P. Hernandez and O. Mena, Nucl. Phys. B 608, 301 (2001).

[7] H. Minakata and H. Nunokawa, JHEP 0110, 001 (2001).

[8] G. L. Fogli and E. Lisi, Phys. Rev. D 54, 3667 (1996).

[9] V. Barger, D. Marfatia and K. Whisnant, Phys. Rev. D 65, 073023 (2002).

[10] H. Minakata and H. Nunokawa, Phys. Lett. B 413, 369 (1997); V. Barger, D. Marfatia and K. Whisnant, Phys. Rev. D 66, 053007 (2002); V. Barger, D. Marfatia and K. Whisnant, Phys. Lett. B 560, 75 (2003); O. Mena and S. J. Parke, Phys. Rev. D 70, 093011 (2004); O. Mena Requejo, S. Palomares-Ruiz and S. Pascoli, Phys. Rev. D 72, 053002 (2005); M. Ishitsuka, T. Kajita, H. Minakata and H. Nunokawa, Phys. Rev. D 72, 033003 (2005); K. Hagiwara, N. Okamura and K. i. Senda, Phys. Rev. D 76, 093002 (2007).

[11] P. Huber, M. Lindner and W. Winter, Nucl. Phys. B 645, 3 (2002); P. Huber, M. Lindner and W. Winter, Nucl. Phys. B 654, 3 (2003).

[12] A. Donini, D. Meloni and P. Migliozzi, Nucl. Phys. B 646, 321 (2002); D. Autiero et al., Eur. Phys. J. C 33, 243 (2004).

[13] A. Donini, E. Fernandez-Martinez and S. Rigolin, Phys. Lett. B 621, 276 (2005); A. Donini, E. Fernandez-Martinez, D. Meloni and S. Rigolin, Nucl. Phys. B 743, 41 (2006).

[14] S. K. Agarwalla, S. Choubey, S. Goswami and A. Raychaudhuri, Phys. Rev. D 75, 097302 (2007).

[15] P. Huber, M. Maltoni and T. Schwetz, Phys. Rev. D 71, 053006 (2005);

[16] J. E. Campagne, M. Maltoni, M. Mezzetto and T. Schwetz, JHEP 0704, 003 (2007).

[17] P. Huber, M. Lindner, T. Schwetz and W. Winter, Nucl. Phys. B 665, 487 (2003).

[18] P. Huber and W. Winter, Phys. Rev. D 68, 037301 (2003).

[19] A. Y. Smirnov, arXiv:hep-ph/0610198.

[20] M. Freund, M. Lindner, S. T. Petcov and A. Romanino, Nucl. Phys. B 578, 27 (2000).

[21] S. K. Agarwalla, A. Raychaudhuri and A. Samanta, Phys. Lett. B 629, 33 (2005). 
[22] S. K. Agarwalla, S. Choubey and A. Raychaudhuri, Nucl. Phys. B 771, 1 (2007).

[23] S. K. Agarwalla, S. Choubey and A. Raychaudhuri, Nucl. Phys. B 798, 124 (2008).

[24] P. Zucchelli, Phys. Lett. B 532, 166 (2002).

[25] M. S. Athar et al. [INO Collaboration], A Report of the INO Feasibility Study, http://www.imsc.res.in/ ino/OpenReports/INOReport.pdf

[26] C. Rubbia, A. Ferrari, Y. Kadi and V. Vlachoudis, Nucl. Instrum. Meth. A 568, 475 (2006);

C. Rubbia, arXiv:hep-ph/0609235.

[27] Y. Mori, Nucl. Instrum. Meth. A 562, 591 (2006).

[28] M. Mezzetto, J. Phys. G 29, 1771 (2003) [arXiv:hep-ex/0302007]. M. Mezzetto, Nucl. Phys. Proc. Suppl. 143, 309 (2005). M. Mezzetto, Nucl. Phys. Proc. Suppl. 155, 214 (2006);

[29] A. Donini, E. Fernandez-Martinez, P. Migliozzi, S. Rigolin and L. Scotto Lavina, Nucl. Phys. B 710, 402 (2005).

[30] A. Donini, E. Fernandez, P. Migliozzi, S. Rigolin, L. Scotto Lavina, T. Tabarelli de Fatis and F. Terranova, arXiv:hep-ph/0511134; A. Donini, E. Fernandez-Martinez, P. Migliozzi, S. Rigolin, L. Scotto Lavina, T. Tabarelli de Fatis and F. Terranova, Eur. Phys. J. C 48, 787 (2006).

[31] P. Coloma, A. Donini, E. Fernandez-Martinez and J. Lopez-Pavon, JHEP 0805, 050 (2008) arXiv:0712.0796 [hep-ph].

[32] J. Burguet-Castell, D. Casper, E. Couce, J. J. Gómez-Cadenas and P. Hernandez, Nucl. Phys. B 725, 306 (2005).

[33] J. Burguet-Castell, D. Casper, J. J. Gómez-Cadenas, P. Hernandez and F. Sanchez, Nucl. Phys. B 695, 217 (2004).

[34] A. Jansson, O. Mena, S. Parke and N. Saoulidou, arXiv:0711.1075 [hep-ph].

[35] P. Huber, M. Lindner, M. Rolinec and W. Winter, Phys. Rev. D 73, 053002 (2006).

[36] C. Volpe, J. Phys. G 34, R1 (2007).

[37] A. Donini and E. Fernandez-Martinez, Phys. Lett. B 641, 432 (2006).

[38] D. Meloni, O. Mena, C. Orme, S. Palomares-Ruiz and S. Pascoli, arXiv:0802.0255 [hep-ph].

[39] S. K. Agarwalla, S. Choubey and A. Raychaudhuri, AIP Conf. Proc. 981, 84 (2008).

S. K. Agarwalla, S. Choubey and A. Raychaudhuri, AIP Conf. Proc. 939, 265 (2007).

[40] P. Huber, M. Lindner, M. Rolinec and W. Winter, Phys. Rev. D 74, 073003 (2006).

[41] S. K. Agarwalla, S. Choubey, A. Raychaudhuri and W. Winter, JHEP 0806, 090 (2008) arXiv:0802.3621 [hep-ex]. 
[42] M. Lindroos, Talk at Moriond Workshop on "Radioactive beams for nuclear physics and neutrino physics" March 17-22nd, 2003; http://moriond.in2p3.fr/radio/index.html.

[43] M. Lindroos, Talk at the "First plenary meeting of the International Design Study for the Neutrino Factory", RAL, United Kingdom, 16-17 January 2008.

[44] A. M. Dziewonski and D. L. Anderson, Phys. Earth Planet. Interiors 25, 297 (1981);

S. V. Panasyuk, Reference Earth Model (REM) webpage, http://cfauves5.harvrd.edu/lana/rem/index.html.

[45] L. Wolfenstein, Phys. Rev. D 17, 2369 (1978);

[46] S. P. Mikheev and A. Y. Smirnov, Sov. J. Nucl. Phys. 42, 913 (1985) [Yad. Fiz. 42, 1441 (1985)]; S. P. Mikheev and A. Y. Smirnov, Nuovo Cim. C 9, 17 (1986).

[47] V. D. Barger, K. Whisnant, S. Pakvasa and R. J. N. Phillips, Phys. Rev. D 22, 2718 (1980).

[48] M. Freund, P. Huber and M. Lindner, Nucl. Phys. B 615, 331 (2001).

[49] M. Lindroos, arXiv:physics/0312042; M. Lindroos, Nucl. Phys. Proc. Suppl. 155, 48 (2006).

[50] http://beta-beam.web.cern.ch/beta\%2Dbeam/

[51] L. P. Ekstrom and R. B. Firestone, WWW Table of Radioactive Isotopes, database version 2/28/99 from URL http://ie.lbl.gov/toi/ 